\title{
Gute Öffentlichkeitsarbeit und die Europäische Union
}

\author{
Tobias Mast*
}

Wissenschaftlicher Mitarbeiter am Bundesverfassungsgericht, Karlsruhe, Deutschland

tobias.mast@mail.de

$\begin{array}{ll}\text { Abstract } & 443\end{array}$

Keywords 444

I. Kurze Geschichte europäischer Öffentlichkeitsarbeit 444

II. Strukturelle und institutionelle Defizite 447

1. Europäische Öffentlichkeit 448

2. Die kommunizierende Union 449

a) Nachrichtenwerte $\quad 450$

b) Die Organe und ihre spezifischen Kommunikationsbedingungen 452

aa) Europäische Kommission 452

bb) Europäisches Parlament 453

cc) Rat 455

III. Qualität der Öffentlichkeitsarbeit und Demokratieprinzip 456

1. Thematikdimension 460

a) Pflicht zur Öffentlichkeitsarbeit 460

b) Prinzip der begrenzten Einzelermächtigung 462

c) Fehlinformation und Manipulation 463

d) Unverständliches und Verkürzungen 467

2. Verfahrensdimension 469

$\begin{array}{ll}\text { 3. Verantwortlichkeitsdimension } & 472\end{array}$

$\begin{array}{ll}\text { IV. Hindernisse und Begünstigungen } & 474\end{array}$

V. Puzzlestück statt Allheilmittel 477

Summary: Good Public Relations and the European Union $\quad 478$

$\begin{array}{ll}\text { Keywords } & 478\end{array}$

\section{Abstract}

Die Europäische Union (EU) ist kein Staat und gründet sich doch auf die Werte der Demokratie und Rechtsstaatlichkeit. Deswegen erstaunt es, dass sich die Europarechtswissenschaft - anders als etwa die Politikwissenschaft bislang kaum mit der Öffentlichkeitsarbeit der Europäischen Union beschäftigt hat, obgleich sie seit jeher deren demokratische Legitimation in Frage

\footnotetext{
* Dr., der Autor ist wissenschaftlicher Mitarbeiter am Bundesverfassungsgericht (Dezernat BVR Prof. Dr. Henning Radtke).
} 
stellt. Die Öffentlichkeitsarbeit der Europäischen Union unterscheidet sich hinsichtlich ihrer institutionellen und rechtlichen Rahmenbedingungen wesentlich von derjenigen ihrer Mitgliedstaaten. Dieser Beitrag stellt kommunikations- und politikwissenschaftliche Erkenntnisse über die Defizite der aktuellen Öffentlichkeitsarbeit der Europäischen Union dar und arbeitet darauf aufbauend heraus, inwiefern das Primärrecht diesen entgegenwirken kann. Öffentlichkeitsarbeit wird hierbei verstanden als diejenigen öffentlichen Äußerungen und Publikationen von EU-Mitarbeitern, Institutionen und Organen, die sich thematisch auf die Union selbst beziehen und deren Handlungen und Vorgänge der näheren Vergangenheit, Gegenwart und $\mathrm{Zu}-$ kunft sowie langfristige Konzepte vor den Unionsbürgern erläutern und rechtfertigen, unabhängig des Mediums.

\section{Keywords}

Europäische Union - Öffentlichkeitsarbeit - Kommunikation - Demokratieprinzip - Legitimation

\section{Kurze Geschichte europäischer Öffentlichkeitsarbeit}

Auf nationaler Ebene ist Öffentlichkeitsarbeit so alt, wie der Nationalstaat selbst. ${ }^{1}$ Das ist auf supranationaler Ebene anders: Eine Kommunikationsstrategie der Europäischen Union war vor der fehlgeschlagenen Ratifikation des Vertrags von Maastricht kaum vorhanden. ${ }^{2}$ Die öffentliche Meinung war der Europäischen Union zwar nicht egal: Seit 1973 führte die Europäische Kommission regelmäßig das Eurobarometer, eine Reihe von öffentlichen Meinungsumfragen, durch. Ein Hauptziel bestand seit jeher darin, die Euro-

1 Helmut Quaritsch, Vorwort des Tagungsleiters, in: Helmut Quaritsch (Hrsg.), Die Selbstdarstellung des Staates, Berlin: Duncker \& Humblot 1977, 3-8 (3); Udo Di Fabio, Information als hoheitliches Gestaltungsmittel, JuS 1997, 1-7.

2 Vgl. Christoph O. Meyer, Political Legitimacy and the Invisibility of Politics, J. Common Mkt. Stud. 37 (1999), 617-639 (623 ff.); Marc R. Gramberger, Die Öffentlichkeitsarbeit der Europäischen Kommission 1952-1996. PR zur Legitimation von Integration?, Baden-Baden: Nomos 1997, 274; Chiara Valentini, The Public Relations of the European Union: New Challenges in a More Integrated Europe, Ninth Annual International Public Relations Research Conference "Changing Roles and Functions in Public Relations", 9.-12.3.2006, Miami, Florida, USA, 2006, 1-21 (6f.); Alice Srugies, Competition or Cooperation? A Comparative Analysis of the Public Diplomacy of the European Union and Selected Member States, ebook, urn:nbn:de: gbv:ilm1-2016000347, 2016, 109 ff.; Michael Brüggemann, Europäische Öffentlichkeit durch Öffentlichkeitsarbeit, Berlin/Heidelberg: Springer 2008, $122 \mathrm{f}$. 
päer zu befragen, ob sie der Europäischen Union vertrauen und ein positives Bild von ihr haben. ${ }^{3}$ Man befürchtete allerdings in den Anfangsjahren, dass gesteigerte öffentliche Aufmerksamkeit einer weiteren Integration eher abträglich sein könnte (permissive consensus) ${ }^{4}{ }^{5}$

Dies änderte sich, nachdem das Ratifizierungsverfahren des Vertrags von Maastricht im Jahr 1992 aufgrund von Widerständen in einigen Mitgliedstaaten unerwartet beschwerlich ausfiel. Hiernach wurde erstmals eine Strategie für Informationspolitik entwickelt, die auf demokratische Legitimationsförderung ausgerichtet war. ${ }^{6}$ Während für die anschließenden 90er- und frühen 00er-Jahre die Öffentlichkeitsarbeit noch als sehr amateurhaft beschrieben wird, ${ }^{7}$ hat sich die Palette der Kommunikationsinstrumente seit der Jahrtausendwende diversifiziert und das Verhältnis der Union zu den Medien professionalisiert. ${ }^{8}$ Auch die inzwischen $\mathrm{zu}$ hunderten, von der Europäischen Kommission errichteten Europe-Direct-Informationszentren und Europäischen Dokumentationszentren verdeutlichen diese Anstrengungen.

Seither wurden von der Europäischen Kommission zahlreiche Papiere und Strategien veröffentlicht, die Ausrichtung der Öffentlichkeitsarbeit wechselte über die Jahre mehrmals, ohne jedoch tatsächlich nennenswerte Erfolge zu verzeichnen. Mit der Informations- und Kommunikationsstrategie für die Europäische Union ${ }^{9}$ wurde im Jahr 2002 ein Paradigmenwechsel hin zu einer aktiveren und persuasiveren Informationspolitik erklärt. ${ }^{10}$ Der 2005 unter Kommissarin Margot Wallström veröffentlichte Aktionsplan für eine bessere

3 Siehe den aktuellen Standard-Eurobarometer 94, für den Daten zwischen Februar und März 2021 erhoben wurden: <https://ec.europa.eu>. Kritisch zu dem Eurobarometer Martin Höpner/Bojan Jurczyk, Kritik des Eurobarometers: Über die Verwischung der Grenze zwischen seriöser Demoskopie und interessengeleiteter Propaganda, Leviathan 40 (2012), 326-349.

4 Leon N. Lindberg/Stuart A. Scheingold, Europe's Would-Be Polity: Patterns of Change in the European Community, Englewood Cliffs, N. J.: Prentice-Hall 1970, $38 \mathrm{ff}$.

5 Meyer (Fn. 2), 624; vgl. Brüggemann (Fn. 2), 120 f.; Rachid Azrout/Jost Van Spanje/Claes De Vreese, When News Matters: Media Effects on Public Support for European Union Enlargement in 21 Countries, J. Common Mkt. Stud. 50 (2012), 691-708.

6 Commission (1993): Information and Communication Policy, Communication of $\mathrm{Mr}$ Pinheiro to the Commission, SEC (93) 916/9. Brussels; Gramberger (Fn. 2), 250.

7 Markus Thiel, European Public Spheres and the EU's Communication Strategy - From Deficits to Policy Fit, Perspectives on European Politics and Society 9 (2008), 342-356; vgl. Sylke. Nissen, Kommunikation in der Krise, ZfP 57 (2010), 453-473 (457 f.).

8 Brüggemann (Fn. 2), 283 f.; Nissen (Fn. 7), 457 f.; Josef Mantl, Transparenz und Kommunikation der Europäischen Union im Lichte des Art 15 AEUV, Wien: Böhlau 2016, 172 ff.; Christoph O. Meyer, Does European Union Politics Become Mediatized?, in: Journal of European Public Policy 16 (2009), 1047-1064 (1059f.).

9 Information and Communication Strategy for the European Union, $\operatorname{COM}(2002) 350$ final.

10 Brüggemann (Fn. 2), 142; Nissen (Fn. 7), 458 f. 
Kommunikationsarbeit der Kommission zu Europa ${ }^{11}$ forderte die Organe auf, sich zu koordinieren und am Informationsinteresse der Unionsbürger auszurichten. Eine Qualitätssicherung sollte etabliert und lokale Zielgruppen adressiert werden. ${ }^{12}$ Auch wurde bemängelt, dass bislang meist nachträglich über abgeschlossene Vorgänge berichtet werde. ${ }^{13}$ Mit dem im Anschluss erarbeiteten Weißbuch über eine europäische Kommunikationspolitik ${ }^{14}$ richtete sich die Kommission stärker auf die Prämissen des Dialogs und der öffentlichen Debatte aus. Erstmals versuchte sie auch das Europäische Parlament und die Regierungen miteinzubeziehen, um eine einheitliche „europäische“ Informationspolitik zu entwerfen. Bewusst wählte man das auf Konsultation der involvierten Akteure ausgerichtete Format eines Weißbuchs. ${ }^{15}$ Auf einer Linie hiermit liegt die 2007 getroffene Interinstitutionelle Vereinbarung Partnerschaft für die Kommunikation über Europa ${ }^{16}$ mit der bessere Absprachen zwischen der Kommission, dem Europäischen Parlament und den Mitgliedstaaten unter Beibehaltung grundsätzlicher Autonomie der Informationspolitik vereinbart wurden. ${ }^{17}$ Parlament, Rat und Kommission erließen 2009 eine entsprechende Erklärung „Europa partnerschaftlich kommunizieren“ ${ }^{18}$ wodurch die bereits zuvor existierende, aber mit wenig Autorität ausgestattete ${ }^{19}$ Interinstitutionelle Gruppe Information (IGI) bekräftigt werden sollte.

An kommunikationspolitischem Willen mangelte es demnach nicht. Allein an der Umsetzung haperte es. Seit dem Jahr 2009 existiert das Amt des Kommissars für Kommunikationsstrategie nicht mehr. Die Thematik ist nunmehr bei der Generaldirektion Kommunikation, ${ }^{20}$ einer Dienststelle der Kommission, angesiedelt. Sie legt nach wie vor Strategien fest und überwacht diese - allerdings nur für die Kommission. Die Zeit der vollmundigen Papiere und Aktionspläne scheint deswegen vorerst vorbei. Seit einigen Jahren lässt sich aber beobachten, dass die Europäische Union auf die öffentliche Meinung weniger durch Experten und Technokraten, sondern auch stärker polifinal

11 Action Plan to Improve Communicating Europe by the Commission, SEC(2005) 985

12 Brüggemann (Fn. 2), 144 f.; Mantl (Fn. 8), 177 ff.

13 Meyer (Fn. 8), 1057.

14 White Paper on a European Communication Policy, COM(2006) 35 final.

15 Brüggemann (Fn. 2), 146, 285; Nissen (Fn. 7), 460 f.; Mantl (Fn. 8), 185 ff.

16 Inter-Institutional Agreement on Communicating Europe in Partnership, COM(2007) 569 final.

17 Brüggemann (Fn. 2), 147; Mantl (Fn. 8), $191 \mathrm{ff.}$

18 2009/C 13/02, abgedruckt in der Geschäftsordnung des Europäischen Parlaments 2011 Anlage XIX.

19 Brüggemann (Fn. 2), 138.

20 Siehe etwa den Strategic Plan 2016-2020* DG COMMUNICATION (Ref. Ares(2016) 1853065); vgl. Irena A. Szyszko, Bürgergemeinschaft oder postnationale Bürokratie?, Münster: LIT 2014, $349 \mathrm{ff}$. 
tisierend einzuwirken versucht. ${ }^{21}$ Prägend dürfte das Jahr 2005 gewesen sein, in welchem die Referenden in Frankreich und den Niederlanden zu dem geplanten europäischen Verfassungsvertrag negativ ausfielen, aber auch das Jahr 2008 mit dem zunächst negativen Referendum Irlands zu dem im Anschluss ausgearbeiteten Vertrag von Lissabon. ${ }^{22}$ Auch das Aufsteigen euroskeptischer Parteien und der Austritt Großbritanniens fordern Gegenreaktionen auf europäischer Ebene heraus. ${ }^{23}$

Auf die Umsetzung der Kommunikationsstrategien wirkt das Selbstverständnis der Unionsverwaltung ein. Aufschlussreich sind insoweit der Kodex für gute Verwaltungspraxis in den Beziehungen der Europäischen Kommission zur Öffentlichkeit ${ }^{24}$ und der Europäische Kodex für gute Verwaltungspraxis des Europäischen Bürgerbeauftragten. ${ }^{25}$ Vor allem letzterer erinnert an die Rechtsgebundenheit der Verwaltungsbeamten und deren öffentliche Vertrauensposition. Er fordert daher ein Gefühl des Anstands, eine glaubwürdige Kontroll- und Fehlerkultur sowie ein höfliches und verständliches Auftreten nach außen.

\section{Strukturelle und institutionelle Defizite}

Einige Gründe dafür, dass die Öffentlichkeitsarbeit der Europäischen Union trotz dieser nun 20 Jahre währenden Anstrengungen nicht als Erfolg

21 Markus Haverland/Minou de Ruiter/Steven. Van de Walle, Agenda-Setting by the European Commission. Seeking Public Opinion?, Journal of European Public Policy 25 (2018), 327-345 (328 ff.).

22 Frank Schorkopf, Der Europäische Weg, 2. Aufl., Tübingen: Mohr Siebeck 2015, $142 \mathrm{ff}$.

23 M. Haverland/M. de Ruiter/S. Van de Walle (Fn. 21), 323; vgl. Christoph Möllers/Linda Schneider, Demokratiesicherung in der Europäischen Union, Tübingen: Mohr Siebeck 2018, $139 \mathrm{ff}$.

24 Anhang, $13 \mathrm{ff}$.

$25 \mathrm{Zu}$ den letzten beiden Kodizes Knut Bourquain, Die Förderung guten Verwaltungshandelns durch Kodizes, DVBl 123 (2008), 1224-1233 (1231 ff.); Julia Haas, Der Ombudsmann als Institution des Europäischen Verwaltungsrechts, Tübingen: Mohr Siebeck 2012, 263 ff.; Martina Lais, Das Recht auf eine gute Verwaltung unter besonderer Berücksichtigung der Rechtsprechung des Europäischen Gerichtshofs, ZEuS 2002, 447-482 (476); Paivi Leino, The Wind is in the North: the first European Ombudsman (1995-2003), European Public Law 10 (2004), 333-368 (349f.); Magdalena E. de Leeww, The European Ombudsman's Role as a Developer of Norms of Good Administration, European Public Law 17 (2011), 349-368; José. Martinez Soria, Die Kodizes für gute Verwaltungspraxis, EuR 36 (2001), 682-705; Julio Ponce Solé, Good Administration and European Public Law, ERPL 14 (2002), 1503-1544 (1529ff.); Kristin Pfeffer, Das Recht auf eine gute Verwaltung, Baden-Baden: Nomos 2006, 55 ff.; Jill Wakefield, Right to Good Administration, Alphen aan den Rijn: Kluwer Law International 2007, 43 ff.; Annette Guckelberger, Der Europäische Bürgerbeauftragte, Berlin: Duncker \& Humblot 2003, $107 \mathrm{ff}$. 
gehandelt wird, liegen in der Struktur des Kommunikationsraums und im institutionellen Zuschnitt der Europäischen Union.

\section{Europäische Öffentlichkeit}

Die Europäische Union ist ein eigenartiger Kommunikationsraum. Ein interdisziplinärer Evergreen ist die Frage, ob eine Europäischen Öffentlichkeit im Sinne einer gemeinsamen medialen und öffentlichen Sphäre überhaupt existiert. Aus normativer und empirischer Perspektive behandelt, ${ }^{26}$ wird diese oftmals in Frage gestellt und eher das Bild allenfalls vernetzter Nationalöffentlichkeiten bemüht. Die strukturelle Schwäche einer Europäischen Öffentlichkeitssphäre liegt dabei in der sprachlichen und kulturellen Segmentierung und dem Mangel paneuropäischer Medien begründet. ${ }^{27}$ Das Bundesverfassungsgericht (BVerfG) stellte in seinem Lissabon-Urteil entsprechend fest: „Auch wenn durch die großen Erfolge der europäischen Integration eine gemeinsame und miteinander im thematischen Zusammenwirken

26 Aus juristischer Perspektive Peter Häberle, Gibt es eine europäische Öffentlichkeit?, Oldenburg: de Gruyter 2000; Claudio Franzius, Europäische Öffentlichkeit und europäische Verfassung, KritVj 86 (2003), 325-339; Anne Peters, Europäische Öffentlichkeit im europäischen Verfassungsprozess, EuR 39 (2004), 375-392; Stephan Bredt, The European Social Contract and the European Public Sphere, ELJ 12 (2006), 61-77 (68 ff.); Claudio Franzius/Ulrich K. Preuß, Die Zukunft der europäischen Demokratie, Baden-Baden: Nomos 2012, 37 ff., 123 ff.; Schorkopf (Fn. 22), $141 \mathrm{ff}$.; aus sozialwissenschaftlicher, empirischer und philosophischer Perspektive Klaus Eder/Kai-Uwe Hellmann/Hans-Jörg Trenz, Regieren in Europa jenseits öffentlicher Legitimation? Eine Untersuchung zur Rolle von politischer Öffentlichkeit in Europa, in: Beate Kohler-Koch (Hrsg.), Regieren in entgrenzten Räumen, PVS Sonderheft 19/1998, 321344; Jürgen Habermas, Warum braucht Europa eine Verfassung?, DIE ZEIT 27/2001; Catbleen Kantner, Kein modernes Babel. Kommunikative Voraussetzungen europäischer Öffentlichkeit, Wiesbaden: Springer VS 2004; Rund Koopmans/Jessica Erbe, Towards a European Public Sphere?, Innovation: The European Journal of Social Science Research 17 (2004), 97-118; HansJörg Trenz, Europa in den Medien, Frankfurt/New York: Campus 2005, 55 ff.; Luuk van Middelaar, Vom Kontinent zur Union, Berlin: Suhrkamp 2016, $345 \mathrm{ff} ., 466 \mathrm{ff} . ;$ Brüggemann (Fn. 2), 58 ff.; Lance Bennett, Grounding the European Public Sphere, KFG Working Paper 43 (2012); Mayte Peters, The Democratic Function of the Public Sphere in Europe, GLJ 14 (2013), 673-693 (680 ff.); Hannab Lorenz, Im Spannungsfeld von Wollen, Sollen und Können - Brüsseler Agenturjournalisten als Nachrichtendienstleister, Baden-Baden: Nomos 2017, 152 ff.; Jørgen Bølstad, Dynamics of European Integration: Public Opinion in the Core and Periphery, European Union Politics 16 (2014), 23-44 (38) spricht auf empirischer Grundlage von einer „common European mood for integration“.

27 Peter Graf Kielmannsegg, Integration und Demokratie, in: Markus Jachtenfuchs/Beate Kohler-Koch (Hrsg.), Europäische Integration, 2. Aufl., Berlin/Heidelberg: Springer Verlag 2003, $49-76$ (58 ff.); Brüggemann (Fn. 2), 33 f.; vgl. Dimiter Toshkov, Public Opinion and Policy Output in the European Union: A Lost Relationship, European Union Politics 12 (2011), 169191 (172). 
stehende europäische Öffentlichkeit in ihren jeweiligen staatlichen Resonanzräumen ersichtlich wächst [...], so ist doch nicht zu übersehen, dass die öffentliche Wahrnehmung von Sachthemen und politischem Führungspersonal in erheblichem Umfang an nationalstaatliche, sprachliche, historische und kulturelle Identifikationsmuster angeschlossen bleibt.“" ${ }^{28}$ In den letzten Jahren erhielt das Forschungsfeld einen neuen Impuls durch die vermehrte Internetkommunikation der Unionsbürger. ${ }^{29}$ Auch die Organe der Europäischen Union nutzen seit einigen Jahren sämtliche gängigen Social MediaPlattformen für ihre Öffentlichkeitsarbeit, ${ }^{30}$ sodass sie unabhängiger von klassischen Massenmedien werden (Disintermediation). ${ }^{31}$ Einer Europäischen Öffentlichkeit mag das förderlich sein, bislang bleiben die fundamentalen Wandlungsprozesse allerdings aus.

\section{Die kommunizierende Union}

Ein Faktor europäischer Öffentlichkeit könnte die Europäische Union selbst sein, die ihr Ziel der „Verwirklichung einer immer engeren Union der Völker Europas“ prominent in Art. 1 Abs. 2 Vertrag über die Europäische Union (EUV) führt. Durch Gesetzgebung, Verwaltung und Rechtsprechung trägt sie maßgeblich zu ihrem Image bei. Hierfür müssen die Unionsbürger aber zunächst einmal von diesen Maßnahmen, den dahinterstehenden Zielen und Idealen erfahren. Eine breitenwirksame Öffentlichkeitsarbeit der Europäischen Union könnte einen wichtigen Beitrag dazu leisten. Allerdings zeichnet ein Blick in die kommunikationswissenschaftliche Fachliteratur

28 BVerfGE 123, 267 (359) - Lissabon.

29 Zur Rolle des Internets und insb. von Social Media für die Bildung einer europäischen Öffentlichkeit Asimina Michailidou, The Role of the Internet in the European Union's Public Communication Strategy and the Emerging European Public Sphere, Loughborough University, Thesis, 2006 <https://hdl.handle.net/2134/3055>; Franzius/Preuß (Fn. 26), 127 f.; Michael Bossetta/Anamaria Dutceac Segesten/ Hans-Jörg Trenz, Engaging with European Politics Through Twitter and Facebook: Participation Beyond the National?, in: Mauro Barisione/ Asimina Michailidou (Hrsg.), Social Media and European Politics, Camden: Palgrave Macmillan 2017, 53-76; M. Peters (Fn. 26), 684 ff., 691 f.; zu internetbedingten Gefahren für den öffentlichen Diskurs auf europäischer Ebene Jens Brauneck, EU-Desinformationsbekämpfung durch Google, Facebook u. a. unter Androhung von Gesetzen, EU-Außenpolitik durch Gegenpropaganda in Drittstaaten?, EuR 55 (2020), 89-111.

30 Michal Krzyżanowski, Social Media in/and the Politics of the European Union, Journal of Language and Politics 17 (2018), 281-304 (passim).

31 Generell Robert Gellman, Disintermediation and the Internet, Government Information Quarterly 13 (1996), 1-8; Axel Bruns, Vom Gatekeeping zum Gatewatching, in: Christoph Neuberger/Christian Nuernbergk/Melanie Rischke (Hrsg.), Journalismus im Internet, Wiesbaden: Springer VS 2009, 107-128. 
schnell ein gegenteiliges Bild: Der Europäischen Union wird insgesamt ein Kommunikationsdefizit nachgesagt, wobei die Institutionen unter grundlegend unterschiedlichen Bedingungen kommunizieren und ihre Bedeutung von Medienschaffenden und der Öffentlichkeit unterschiedlich wahrgenommen wird. ${ }^{32}$ Sie alle eint, dass sie die Nachrichtenwerte, die entscheidend für ein mediales Aufgreifen und die spätere Rezeption sind, nicht optimal bespielen.

\section{a) Nachrichtenwerte}

Nachrichtenwerte beschreiben Kriterien, welche die Auswahl und Präsentation von Ereignissen durch Journalisten sowie die spätere Rezeptionschance beeinflussen. ${ }^{33}$ Aus der Vielzahl der relevanten Faktoren versagt die Europäische Union dabei vor allem, die folgenden zu bespielen: Einfachheit, Konflikt, Personalisierung, Nähe und kurze Ereignisdauer. ${ }^{34}$ Augenfällig wird das zunächst für die Kriterien der Nähe und des Konflikts. In ihrem Mehrebenensystem findet die Politik räumlich und zeitlich weit entfernt von den Bürgern statt, an einem Ort, an dem Parteien nicht um Regierungsämter konkurrieren und keine Opposition Gegenvorschläge unterbreitet. ${ }^{35}$ Wenn häufig Jahre ins Land ziehen, bis Initiativen und Entscheidungen der Europäischen Union auf nationaler Ebene - dann unter größerer Beachtung der Öffentlichkeit - umgesetzt und angewendet werden, fällt es schwer, deren Nähe und Relevanz zu vermitteln. ${ }^{36}$ Zudem gelingt es mitunter nicht, Handlungsabläufe zu erkennen, das Geschehen zu personifizieren, es auf einen zweiseitigen Konflikt einzuengen, es in dem institutionellen Geflecht ver-

32 Ana I. Martins/Sophie Lecheler/Claes. H. de Vreese, Information Flow and Communication Deficit: Perceptions of Brussels-Based Correspondents and EU Officials, Journal of European Integration 34 (2012), 305-322 (305 f., 317); Bo Laursen, Transparency in the Council of the European Union: Why Journalists Don't Get the Full Picture, Journalism 14 (2012), 771 789 (773); Meyer (Fn. 2), 617 ff.

33 Klassisch Walter Lippmann, Public Opinion, Harcourt: San Diego California 1922, Chapter XXIII; Winfried Schulz, Die Konstruktion von Realität in den Nachrichtenmedien, Freiburg (Breisgau): Alber 1976, $11 \mathrm{ff}$.; Einar Østergaard, Factors Influencing the Flow of News, JPR 2 (1965), 39-63; Johan Galtung/Mari H. Ruge, The Structure of Foreign News, JPR 2 (1965), 64-90; Christiane Eilders/Werner Wirth, Die Nachrichtenwertforschung auf dem Weg zum Publikum, Publizistik 44 (1999), 34-57.

34 Brüggemann (Fn. 2), 31 f.; vgl. Nikolaus Blome/Bernd Ulrich, Europa als Auflagenkiller - Erfahrungen zweier Journalisten, in: Ingolf Pernice/Lars S. Otto (Hrsg.), Europa vermitteln im Diskurs, Baden-Baden: Nomos 2011, 81-89 (84); Lorenz (Fn. 26), 174.

35 Brüggemann (Fn. 2), 31 ff.; vgl. Blome/Ulrich (Fn. 34), 81 (83); Lorenz (Fn. 26), 173 f.

36 Meyer (Fn. 8) 1055; Gerhard Sabathil, Von der Vielstimmigkeit zum Chor - Kommunikation in Europa, in: Pernice/Otto (Fn. 34), 67 (70). 
ständlich zu verorten und die technischen Einzelheiten in einer Sprache zu formulieren, die für ein großes Publikum zugänglich und attraktiv ist. ${ }^{37}$ Schließlich machen es die hohen Schwellenwerte, die für die Entscheidungsfindung in der Europäischen Union erforderlich sind (viele Institutionen, qualifizierte Mehrheit oder Einstimmigkeit im Rat), für die involvierten Akteure riskant, Kontroversen zu schüren und damit Blockaden politischer Initiativen zu provozieren - Anreize für die EU-Institutionen, sich gegenseitig scheitern $\mathrm{zu}$ sehen, gibt es wenige. ${ }^{38}$ Aber auch die Kriterien der Einfachbeit und Personalisierung werden regelmäßig verfehlt: Die Kommunikation der Europäischen Union wird durch einen Mangel an hoher und positiver Medienpräsenz, den polyphonen Charakter der EU-Kommunikation, die Komplexität des supranationalen Gemeinwesens und das Fehlen einer klaren institutionellen Identität erschwert. ${ }^{39}$ Und selbst wenn Europapolitiker charismatisch und telegen sind, werden sie ihren Charme kaum in den 24 Amtssprachen der Union spielen lassen können. ${ }^{40}$ Auch an intrinsischen Anreizen der einzelnen Akteure an starker Diskurspräsenz könnte es mangeln, denn die Mobilisierung der Unionsbürger ist für die Arbeitsweise der EU-Organe von tendenziell geringerer Bedeutung als auf der Ebene der Mitgliedstaaten. ${ }^{41}$

Es muss auch bedacht werden, was all dies für öffentlich ausgetragene Konflikte zwischen Organen der Europäischen Union und Nationalstaaten bedeutet: Die öffentliche Sichtbarkeit europäischer Themen unterscheidet sich von Staat zu Staat, je nach den nationalen Gegebenheiten und Interessen. ${ }^{42}$ Die Ausgangsposition der Europäischen Union bei Konflikten mit Mitgliedstaaten, die im nationalen öffentlichen Raum ausgetragen werden, ist dabei stets prekär. Zumindest ein Teil der Medien wird aufgrund ausgeprägterer Vernetzung die nationale Regierung unterstützen, EU-Institutionen

37 Jan Beyers/Bart Kerremans, The Press Coverage of Trade Issues: a Comparative Analysis of Public Agenda-Setting and Trade Politics, Journal of European Public Policy 14 (2007), 269292 (272); Vgl. Meyer (Fn. 2), 633.

38 Vgl. Meyer (Fn. 8), 1047 (1058); Sebastiaan Princen, Agenda-Setting in the European Union: a Theoretical Exploration and Agenda for Research, Journal of European Public Policy 18 (2011), 927-943 (940).

39 Chiara. Valentini, Political Public Relations in the European Union: EU Reputation and Relationship Management Under Scrutiny, Public Relations Journal 7 (2013), 2-22 (8); Vgl. Meyer (Fn. 2), 626 f.; Brüggemann (Fn. 2), $28 \mathrm{ff}$.

40 Meyer (Fn. 8), 1055; er weist auch darauf hin, dass aus diesen Gründen selbst im Action Plan to Improve Communicating Europe by the Commission (SEC(2005) 985 final) eine aktive Kommunikation der EU-Vertreter vor allem mit dem eigenen Herkunftsland befürwortet wird; kritisch zu einer solchen nationalen Orientierung M. Peters (Fn. 26), 690.

41 Princen (Fn. 38), 940.

42 Vgl. Beyers/Kerremans (Fn. 37), 273 f. 
haben diesen Heimvorteil nicht. ${ }^{43}$ Im Gegenteil deuten Forschungsergebnisse darauf hin, dass die europäische Politik in den nationalen Medien tendenziell verkürzt oder verzerrt dargestellt wird. ${ }^{44}$

\section{b) Die Organe und ihre spezifischen Kommunikationsbedingungen}

Ein Blick auf die Kommunikationsbedingungen der Kommission, des Parlaments und des Rates als den drei herausragenden politischen Organen der Europäischen Union verdeutlicht die - unterschiedlich stark ausgeprägte Misere.

aa) Europäische Kommission

Obwohl die Europäische Kommission nicht öffentlich tagt, ${ }^{45}$ hat ihre Öffentlichkeitsarbeit eine noch vergleichsweise gute Ausgangsposition. In der Kommunikationswissenschaft wird ihr als einzigem Unionsorgan eine proaktive Informationsstrategie zur Imagepflege attestiert. ${ }^{46}$ Aufgrund ihrer Hybridfunktion mit politischen (Art. 17 Abs. 1 S. 1, Abs. 2 EUV) und Administrativaufgaben (Art. 17 Abs. 1 S. 5 EUV) kommuniziert die Kommission auch nach zwei entsprechenden Logiken: Neben Bewerbung und Rechtfertigung des eigenen Handelns treten sachlichere Erklärungen. ${ }^{47}$ Durch ihr Initiativrecht zur Gesetzgebung ist sie in der Lage, Ereignisse mit hohem Nachrichtenwert zu inszenieren. In dieser Funktion hat die Kommission ein genuines Interesse an der öffentlichen Förderung ihrer politischen Agenda, sie braucht die Unterstützung der nationalen Regierungen für ihre Vorschläge. ${ }^{48}$ Aber auch als „Hüterin der Verträge“ (Art. 17 Abs. 1 S. 3 EUV) bespielt sie den zentralen Nachrichtenwert des Konflikts gegenüber Mitgliedstaaten. Die Besetzung mit vergleichsweise wenigen Kommissaren (Art. 17 Abs. 4 EUV) über eine kontinuitätsstiftende Amtszeit von fünf Jahren (Art. 17 Abs. 3 EUV) bedient mediale Personalisierungstendenzen. ${ }^{49}$ Zudem sind die Vertreter der Kommission im Gegensatz zu den

43 Vgl. Meyer (Fn. 2), 634.

44 Patrick Bijsmans/Christina Altides, 'Bridging the Gap' Between EU Politics and Citizens? The European Commission, National Media and EU Affairs in the Public Sphere, Journal of European Integration. 29 (2007), 323-340 (337 f.).

45 Art. 9 Geschäftsordnung der Kommission (C(2000) 3614).

46 Valentini (Fn. 39), 8; vgl. Steffen B. Rasmussen, The Messages and Practices of the European Union's Public Diplomacy, The Hague Journal of Diplomacy 5 (2010), 263-287 (272f.).

47 Vgl. Brüggemann (Fn. 2), 134.

48 Valentini (Fn. 39), 6.

49 Skeptischer Meyer (Fn. 8), 1055. 
Mitgliedern des Rates vollkommen unabhängig gegenüber den Mitgliedstaaten (vgl. Art. 17 Abs. 3 UAbs. 2, 3 EUV, Art. 245 Abs. 1 Vertrag über die Arbeitsweise der Europäischen Union (AEUV)), sodass Zuordnungsverwirrungen vermieden werden. ${ }^{50}$ Trotz alldem wird kritisiert, die Informationstätigkeit erfolge oftmals in Form von „Pseudo-Dialogen“, die nicht direkt mit dem politischem Entscheidungsprozess verbunden seien und mangels direkter (Ab-)Wählbarkeit der Kommission durch Unionsbürger nicht sanktioniert werden könnten - wahrer demokratischer Legitimation sei die Öffentlichkeitsarbeit damit nur bedingt förderlich. ${ }^{51}$ Sie sei „politisch korrekt, aber wirkungslos". 52

\section{bb) Europäisches Parlament}

Das Parlament kann innerhalb der Europäischen Union vorhandene Informationen katalysieren und im Wege der öffentlichen Tagung (Art. 15 Abs. 2 AEUV) einer breiteren Öffentlichkeit zugänglich machen. ${ }^{53}$ Sein Informationsrecht umfasst dabei auch laufende Deliberationsprozesse in sensiblen Bereichen, etwa den Abschluss internationaler Übereinkünfte betreffend die Gemeinsame Außen- und Sicherheitspolitik (GASP) gemäß Art. 218 Abs. 10 AEUV. ${ }^{54}$ Das Parlament stellt das einzige unmittelbar demokratisch legitimierte Organ der Europäischen Union dar (Art. 14 Abs. 3 EUV), weswegen die politischen Parteien sowie Abgeordnete und solche die es werden wollen ein intrinsisches Interesse haben, insbesondere in zeitlicher Nähe zu Wahlen öffentlich sichtbar zu sein. ${ }^{55}$ Dementsprechend wird empirisch seit der ersten Direktwahl des Parlaments im Jahr 1979 eine etwas aktivere Informationspolitik des Europäischen Parlaments diagnostiziert. ${ }^{56}$ In der Praxis spricht das Parlament typologisch mit „drei Stimmen“:

50 Aufgrund der Neutralität wird ihr aber auch übertriebene Vorsicht in politischen Debatten nachgesagt, Meyer (Fn. 8), 1054.

51 Brüggemann (Fn. 2), 287 f.; vgl. Stijn Smismans, European Civil Society: Shaped by Discourse and Institutional Interests, ELJ 9 (2003), 473-495 (490 ff.); M. Peters (Fn. 26), 678.

52 Brüggemann (Fn. 2), 279.

53 Hervorzuheben sind aus jüngerer Vergangenheit die parlamentarischen Debatten um die Transatlantische Handels- und Investitionspartnerschaft (TTIP) und die Datenschutzgrundverordnung.

54 EuGH, Urteil v. 14.6.2016, Rs. C-263/14, ECLI:EU:C:2016:435 Rn. 68 ff. - Parlament/ Rat betreffend das EU-Tansania-Abkommen.

55 Brüggemann (Fn. 2), 131; vgl. van Middelaar (Fn. 26), 358 f.

56 Ana L. Terra, From Information Policy to Communication Policy: First Steps Towards Reaching European Citizens in the 1970 s and the 1980 s, in: Chiara Valentin/Giorgia Nesti (Hrsg.), Public Communication in the European Union: History, Perspectives and Challenges, Cambridge: Cambridge Scholars Publishing 2010, 49-66 (62). 
einer übergeordneten Institutionsstimme, einer Fraktionsstimme und der Stimme des einzelnen Abgeordneten ${ }^{57 .}{ }^{58}$

Zumindest was die übergeordnete Institution des Parlaments anbelangt, sind die Ausgangsbedingungen für Öffentlichkeitsarbeit allerdings defizitär. Zwar dürften die ursprünglich geäußerten Befürchtungen, ohne Gesetzesinitiativrecht bestünde ein geringerer Anreiz für das Parlament, öffentliche Zustimmung zu suchen, ${ }^{59}$ aufgrund der zwischenzeitlichen Änderungen ${ }^{60}$ des Aufforderungsrechts gegenüber der Kommission (Art. 225 AEUV) und des Entwurfsvorlagerechts gegenüber dem Rat und dem Europäischen Rat (Art. 48 Abs. 2, 6 EUV) nicht mehr gleichermaßen gelten. Generell versperrt sich parlamentarische Arbeit aber „von ihrer kompromißsuchenden Verhandlungsnatur und von ihrer mangelnden Personalisierbarkeit her grundlegend den vorherrschenden Vermittlungsstandards der massenmedialen Öffentlichkeit". ${ }^{61}$ Für das Europäische Parlament, das maximal 750 Sitze, verteilt über Parlamentarier aus allen Mitgliedsstaaten (Art. 14 Abs. 2 EUV) aufweist, wird dieser Befund aufgrund seiner außergewöhnlichen Größe und multipler Konfliktlinien verschärft.

Besonders groß wird das öffentliche Interesse in interorganisatorischen Konfliktsituationen sein, welche etwa die Einsetzung eines Untersuchungsausschusses (Art. 226 AEUV) oder die Einbringung eines Misstrauensantrags (Art. 234 AEUV) zur Folge haben. Insgesamt wird das Parlament seinem normativen Anspruch als Zentrum und Motor des Diskurses außerhalb seiner eigentlichen Sitzungsöffentlichkeit nach verbreiteter Meinung aber nicht gerecht. In der Kommunikationswissenschaft wird es nach wie vor mitunter als „The Great Non-Communicator" bezeichnet. ${ }^{62}$ Neuere Entwicklungen rufen

57 Ein aktuelles Beispiel der Öffentlichkeitsarbeit einzelner Abgeordneter bildet die aufwendige YouTube-Produktion „The Nico Semsrott Show“ des gleichnamigen Satirikers und Mitglieds des Europäischen Parlaments.

58 Valentini (Fn. 39), 7.

59 Meyer (Fn. 8), 1053.

60 Vgl. zur inzwischen stärkeren Position des Europäischen Parlaments Joachim Wuermeling, Mehr Kraft zum Konflikt, EuGRZ 31 (2004), 559-562 (562); Franzius/Preuß (Fn. 26), $133 \mathrm{ff}$.

61 Stefan Marschall, Public Relations der Parlamente. Parlamentarisches Kommunikationsmanagement in der modernen Öffentlichkeit, ZParl 30 (1999), 699-715 (713); vgl. Edwin Czerwick, Parlamentarische Politikvermittlung - zwischen „Basisbezug“ und „Systembezug“, in: Ulrich Sarcinelli (Hrsg.), Politikvermittlung und Demokratie in der Mediengesellschaft, Opladen: Westdeutscher Verlag 1989, 253-272 (259); Edwin Czerwick, Parlament und Politikvermittlung, in: Ulrich Sarcinelli (Hrsg.), Politikvermittlung, Stuttgart: Bonn Aktuell 1987, 161183 (164).

62 Peter J. Anderson/Aileen McLeod, The Great Non-Communicator? The Mass Communication Deficit of the European Parliament and its Press Directorate, J. Common Mkt. Stud. 42 (2004), 897-917. 
dagegen ganz andere Kritik hervor: Vor der Abstimmung zur umstrittenen Novellierung der Urheberrechtsrichtlinie twitterte das Parlament etwa als solches werbend für den Novellierungsvorschlag und positionierte sich damit in bedenklicher Weise zugunsten eines Teils der Fraktionen. ${ }^{63}$

cc) Rat

Auch die Öffentlichkeitsarbeit des Rates muss als ambivalent bezeichnet werden. Er ist trotz der Aufwertung des Europäischen Parlaments nach wie vor als Staatenkammer ein bedeutsames Rechtsetzungsorgan der Europäischen Union, seine Ausgangsbedingungen für eine wirksame Öffentlichkeitsarbeit sind allerdings in mehrerer Hinsicht defizitär.

Der Rat ist ein vielstimmiger politischer Akteur, seine Öffentlichkeitsarbeit muss qua institutionellem Zuschnitt unterschiedliche Stimmen zu den vielfältigen und oft gegensätzlichen politischen Positionen der einzelnen Mitgliedstaaten repräsentieren (Art. 16 Abs. 2 EUV). ${ }^{64}$ Die nationalen Interessen und Stimmen versucht das Generalsekretariat des Rates - mit unterschiedlichem Erfolg - zu organisieren und zu moderieren. ${ }^{65}$ Sowohl die Pressesprecher als auch die EU-Ratspräsidentschaft selbst im Rahmen ihrer Pressekonferenz sind darum bemüht, Wogen zu glätten und den kleinsten gemeinsamen Nenner der Mitgliedstaaten in diplomatischer und förmlicher Sprache nach Abschluss der Verhandlungen zu verkünden. ${ }^{66}$ Allerdings wurden die institutionellen Rahmenbedingungen dafür, einen Einblick in den Entscheidungsfindungsprozess und die einzelnen Positionen zu erhalten, seit dem Vertrag von Lissabon deutlich verbessert: Seither tagt der Rat - digital vermittelt über einen Videostream - öffentlich, wenn er über Entwürfe zu Gesetzgebungsakten berät und abstimmt (Art. 16 Abs. 8 EUV, Art. 15 Abs. 2 S. 2 AEUV). Demgegenüber sind die organübergreifenden Gespräche während des Gesetzgebungsverfahrens und die Sitzungen des Vermittlungsausschusses weiterhin nicht öffentlich, auch werden heikle Themen inzwischen eher informell besprochen. ${ }^{67}$ Schließlich wird nach wie vor ein Anreiz darin gesehen,

63 Siehe <https://twitter.com/Europarl_DE/status/1100705082470027264>.

64 Valentini (Fn. 39), 6; Bo Laursen/Chiara Valentini, Media Relations in the Council of the European Union, Journal of Public Affairs 13 (2013), 230-238 (232).

65 Brüggemann (Fn. 2), 131.

66 Laursen/Valentini (Fn. 64), 234 f.; Laursen (Fn. 32), 771 (779ff.).

67 Jean-Paul Jacqué, in: Hans von der Groeben/Jürgen Schwarze/Armin Hatje, Europäisches Unionsrecht, 7. Aufl., Baden-Baden: Nomos 2015, Art. 16 EUV Rn. 54; Christina Ziegenhorn, in: Eberhard Grabitz/Meinhard Hilf/Martin Nettesheim, Das Recht der Europäischen Union, 69. EL 2020, München: C. H. Beck, Art. 16 EUV Rn. 69; Laursen (Fn. 32), 783 f.; Laursen/Valentini (Fn. 64), 236. Außerhalb des primärrechtlichen Transparenzgebots gilt weiterhin Art. 6 Abs. 1 der Geschäftsordnung des Rates: „[...] unterliegen die Beratungen des Rates der Geheimhaltungspflicht, es sei denn, dass der Rat anders entscheidet.“ 
möglichst wenig öffentliche Aufmerksamkeit - und damit in der Regel auch Kritik - zu erzeugen, um Arbeit des konsensorientierten Ministerrats reibungsfrei zu gestalten. ${ }^{68}$

Im Vergleich zum Europäischen Parlament lässt sich der kleinere Rat zwar stärker personalisieren, zu Verwirrungen kann es allerdings aufgrund seiner wechselnden Zusammensetzungen (Art. 16 Abs. 6 EUV) und dem rotierenden Vorsitz (Art. 16 Abs. 9 EUV) kommen, welche die Aufmerksamkeit vor allem temporär im Vorsitzmitgliedstaat steigern dürften. ${ }^{69}$ Zudem steht der Rat vor einem Multifunktionalitätsproblem: Da er der Einbringung mitgliedstaatlicher Interessen dient und sich aus Akteuren zusammensetzt, die der Öffentlichkeit in entsprechender Funktion bekannt sind, fällt es den Rezipienten hier besonders schwer, ihre Äußerungen der Sphäre der Europäischen Union zuzuordnen. Die Medien- und Politikwissenschaft unterscheidet bei der Untersuchung politischer Öffentlichkeitsarbeit häufig bereits nicht maßgeblich zwischen staatlichen und parteipolitischen Akteuren. ${ }^{70}$ Auch das BVerfG und einige Stimmen in der deutschen Rechtswissenschaft gehen von einer entsprechend undifferenzierten Einordnung durch die Rezipienten aus. ${ }^{71}$ Wer einem solchen Bild indifferenter beziehungsweise sämtliche Rollen erwägender Rezeption anhängt, wird wenig optimistisch hinsichtlich einer genuinen Imagebildung des Rates sein.

\section{Qualität der Öffentlichkeitsarbeit und Demokratie- prinzip}

Erörtert man die demokratische Legitimation der Europäischen Union mit den Begriffen der Input- (government by the people) und Output-Legitimation (government for the people), ${ }^{72}$ kann sich Öffentlichkeitsarbeit auf beiden

68 Meyer (Fn. 8), 1054.

$69 \mathrm{Vgl}$. Laursen/Valentini (Fn. 64), 235: „holding the EU presidency is an opportunity for a government to 'get political kudos at home'“.

70 Vgl. Thomas Meyer, Media Democracy, Hoboken (New Jersey): Blackwell 2002; Kurt Imbof/Roger Blum/Heinz Bonfadelli/Otfried Jarren, Demokratie in der Mediengesellschaft, VS Verlag für Sozialwissenschaften, 2006; Patrick Donges/Otfried Jarren, Politische Kommunikation in der Mediengesellschaft, 3. Aufl., VS Verlag für Sozialwissenschaften, 2011; Rainer Lepsius, Vertrauen zu Institutionen, in: Stefan Hradil (Hrsg.), Differenz und Integration: die Zukunft moderner Gesellschaften, Frankfurt/New York: Campus 1997, 291.

71 BVerfGE 138, 102 (118) - Schwesig; vgl. bereits BVerfGE 44, 125 (187) - Öffentlichkeitsarbeit; weitere Nachweise bei Tobias Mast, Staatsinformationsqualität, Berlin: Duncker \& Humblot 2020, 53.

72 Fritz W. Scharpf, Regieren in Europa: effektiv und demokratisch?, Frankfurt/New York, Campus 1999, 16 f., 167 f.; Meyer (Fn. 2), 618 ff.); Laursen (Fn. 32), 774; vgl. Bredt (Fn. 26), 74. 
Ebenen auswirken. Zum einen erfordern die Prozesse politischer Willensbildung und Kontrolle, die turnusmäßig in Wahlen münden, eine hinreichende Informationsgrundlage der Unionsbürger. ${ }^{73}$ Die Bevölkerung benötigt zutreffendes Wissen, unabhängig davon, ob sie ihre Stimmabgabe auf (Miss-) Erfolge vergangener Wahlperioden bezieht (retrospective voting) ${ }^{74}$ oder zukünftige Entscheidungen und Pläne der Funktionsträger für maßgeblich erachtet (promissory representation) ${ }^{75} .{ }^{76}$ Die Öffentlichkeitsarbeit der Europäischen Union kann keine lebendige europäische Öffentlichkeit als Vorbedingung demokratischer Legitimation erzwingen. Europäische Öffentlichkeit kann man nicht „von oben machen“, sondern muss „von unten entstehen“ ${ }^{77}$ Aber eine wesentliche Voraussetzung für die öffentliche Behandlung von EU-Themen ist Öffentlichkeitsarbeit allemal: ${ }^{78}$ Sie nimmt selbst unmittelbar am öffentlichen Diskurs teil und bildet eine wesentliche Grundlage für die mediale Berichterstattung. Auf beiden Wegen versucht sie Informationen zu verbreiten, Aufmerksamkeit für Themen zu wecken und Meinungen zu setzen. ${ }^{79}$ Das plurale Mediensystem, dem diese Aufgabe gemäß Art. 11 Charta der Grundrechte der Europäischen Union $(\mathrm{GrC})$ primär zugewiesen ist, ${ }^{80}$ könnte dies nicht allein gewährleisten. Auch die Medien sind im alltäglichen

73 Vgl. Dieter Grimm, Does Europe Need a Constitution?, in: ELJ 1 (1995), 282-302 (292 ff.); Heidrun Abromeit, Möglichkeiten und Ausgestaltung einer europäischen Demokratie, in: Ansgar Klein/Ruud Koopmans/ Hans-Jörg Trenz /Ludger Klein/Christian Lahusen /Dieter Rucht (Hrsg.), Bürgerschaft, Öffentlichkeit und Demokratie in Europa, Opladen: Leske + Budrich 2003, 31-54; Graf Kielmannsegg (Fn. 27), 58 ff.; Margot Wallström, Communicating Europe - Mission Impossible?, in: Pernice/Otto (Fn. 34), 21-28 (24); Valentini (Fn. 2), $8 \mathrm{f.}$

74 Morris P. Fiorina, Retrospective Voting in American National Elections, New Haven (Connecticut): Yale University Press 1981, passim.

75 Jane Mansbridge, Rethinking Representation, Am. Polit. Sci. Rev. 97 (2003), 515-528 (516).

76 Hierzu insgesamt Stefan Marschall, Lügen und Politik im „postfaktischen Zeitalter“, Aus Politik und Zeitgeschichte 13/2017, 17-22 (19f.); kritisch zu einem ständigen europäischen Meinungsbildungsprozess Dieter Grimm, Europa ja - aber welches? Zur Verfassung der europäischen Demokratie, München: C. H. Beck 2016, 24 f., 92.

77 Schorkopf (Fn. 22), 143 f.

78 Brüggemann (Fn. 2), 38.

79 Vgl. Ulrike Röttger/Joachim Preusse/Jana Schmitt, Grundlagen der Public Relations, Wiesbaden: Springer VS 2014, 83 ff.; Donges/Jarren (Fn. 70), 185 ff.; Brüggemann (Fn. 2), 71 f.; Marcel Haag, in: von der Groeben/Schwarze/Hatje (Fn. 67), Art. 10 EUV, Rn. 14; Mast (Fn. 71), $77 \mathrm{ff} ., 129$.

80 Vgl. jeweils zu Art. 10 EMRK EuGH, Urteil v. 25.7.1991, Rs. C- 288/89, Slg 1991, I4007, ECLI:EU:C:1991:323 Rn. 23 f. - Stichting Collectieve Antennevoorziening Gouda u. a.;; EuGH, Urteil v. 3.2.1993, Rs. C-148/91, Slg 1993, I-487, Rn. 9 f. - Veronica Omroep Organisatie; EuGH, Urteil v. 25.7.1991, Rs. C-353/89, Slg 1991, I-4069, ECLI:EU:C:1991:325, Rn. 30 Niederlande/Kommission; zu Art. 11 GrC EuGH, Urtel v. 22.1.2013, Rs. C-283/11, ECLI:EU: C:2013:28, Rn. 51 f. - Sky/Österreich; Norbert Bernsdorff, in: Jürgen Meyer/Sven Hölscheidt, Charta der Grundrechte der Europäischen Union, 5. Aufl. 2019, Art. 11 Rn. 19. 
Geschäft auf eine Informationsquelle und gewisse Vorselektierungen und Priorisierungen angewiesen, um ihrer Vermittlungsfunktion gerecht werden zu können. ${ }^{81}$ Zwar ist die Europäische Union dafür berüchtigt, dass viele Informationen von Whistleblowern geleakt werden, ${ }^{82}$ Medienvertreter könnten sich dennoch nicht allein auf diese informelle und unplanbare Ressource verlassen. Ihre Berichterstattung wird durch die Öffentlichkeitsarbeit nicht gänzlich determiniert, beide ermöglichen und beeinflussen sich aber doch gegenseitig (Intereffikation). ${ }^{83}$ Was die Output-Legitimation anbelangt, steigert die fortlaufende Sichtbarkeit und Rechtfertigung der europäischen Handlungen und Prozesse die politische Verantwortlichkeit gegenüber den Unionsbürgern und lässt eine Orientierung der Funktionsträger am öffentlichen Interesse wahrscheinlicher werden. ${ }^{84}$ Unabhängig davon, ob der einzelne Akt der Öffentlichkeitsarbeit im Diskurs aufgegriffen wird oder nicht, dürfte bereits das Wissen der Funktionsträger um diese Möglichkeit präventiv gegenüber Machtmissbrauch und einer Ausrichtung an Partikularinteressen wirken.

Öffentlichkeitsarbeit kann daher die Demokratie stärken. Sie kann die Demokratie aber auch schwächen, indem sie Fehldeutungen provoziert, den öffentlichen Meinungsbildungsprozess verzerrt, übermäßig Partei nimmt, zivilgesellschaftliche Kräfte desavouiert oder Abschreckungseffekte auslöst. Der deutsche Diskurs, der von einigen Leitentscheidungen des BVerfG geprägt ist, zeichnet sich durch die Vorstellung einer besonders wirkmächtigen und missbrauchsanfälligen Öffentlichkeitsarbeit aus. ${ }^{85}$ Auch der Europäische Gerichtshof $(\mathrm{EuGH})$ hat zumindest für die Mitgliedsstaaten erkannt, dass hoheitliche Macht auch ohne imperativen Zwang, nämlich durch bloße Infor-

81 Vgl. D. Morgan, British Media and the European Union News: the Brussels News Beat and its Problems, European Journal of Communication 10 (1995), 321-343 (328f.); P. Statham, Making Europe News, Journalism 9 (2008), 398-422 (403f.); Laursen (Fn. 32), 772 f. Die politischen Parteien, denen ebenfalls eine Vermittlungsfunktion im öffentlichen Diskurs zukommt, sind nach der Konzeption der Art. 10 Abs. 4 EUV, Art. 12 Abs. 2 GrC eher darauf ausgelegt, den Willen der Unionsbürger zur Geltung zu bringen als umgekehrt das Handeln der Europäischen Union sichtbar zu machen. In der Interpretation des BVerfG wird dagegen kein Übergewicht einer bestimmten Vermittlungsrichtung herausgestellt. Vgl. BVerfGE 89, 155 (185) - Maastricht; BVerfGE 123, 267 (358) - Lissabon.

82 Vgl. Brüggemann (Fn. 2), 203, 269; Laursen (Fn. 32), 784; Lorenz (Fn. 26), 332 f.

83 Günter Bentele/Tobas Liebert/Stefan Seeling, Von der Determination zur Intereffikation. Ein integriertes Modell zum Verhältnis von Public Relations und Journalismus, in: Günter Bentele/Michael Haller (Hrsg.), Aktuelle Entstehung von Öffentlichkeit. Akteure-StrukturenVeränderungen, Konstanz: UVK 1997, S. 225-250, insbes. $240 \mathrm{ff}$.

$84 \operatorname{Scharpf(Fn.~72),~23,~168;~Brüggemann~(Fn.~2),~} 46$.

85 BVerfGE 44, 125 (174f., 184 f.) - Öffentlichkeitsarbeit; BVerfGE 105, 252 (257, 269) Glykol; BVerfGE 105, 279 (289) - Osho; BVerfGE 136, 323 (332) - Spinner; BVerfGE 138, 102 (109, 115, 118 ff.) - Schwesig; BVerfGE 140, 225 (227) - Rote Karte; BVerfG, NJW 73 (2020), 2096 (2099 f.) - Seehofer. 
mationstätigkeit ausgeübt werden kann. Hier entschied er, dass eine staatliche Werbekampagne für heimische Produkte gegen die Warenverkehrsfreiheit aus Art. 34 AEUV verstoßen kann. ${ }^{86}$

Einen Beitrag zur demokratischen Legitimation der Europäischen Union leistet Öffentlichkeitsarbeit demnach nicht per se, sondern nur sofern sie gewisse Qualitätsstandards einhält. Das BVerfG betont in diesem Zusammenhang, dass eine funktionsfähige Öffentlichkeit die wesentlichen Ziele und Entscheidungsabläufe der Unionsorgane mitsamt der bestehenden Alternativen ständig sichtbar halten müsse. ${ }^{87}$ Diese Anforderungen sind bemerkenswert anschlussfähig an C. O. Meyers Modell qualitativ hochwertiger Öffentlichkeitsarbeit, das in Politikwissenschaft und Kommunikationswissenschaft inzwischen klassisch geworden ist. Er unterscheidet drei Dimensionen, die entscheidend dafür sind, ob öffentliche Partizipation und Verantwortlichkeit tatsächlich durch Öffentlichkeitsarbeit gefördert werden können: Die Thematikdimension (Was wird wie, mit welchen Argumenten diskutiert?), die Verfabrensdimension (An welchem Punkt des Entscheidungsprozesses werden Themen diskutiert und inwiefern kann das Ergebnis noch beeinflusst werden?) und die Verantwortlichkeitsdimension (Wer vertritt was? Wer ist für getroffene Entscheidungen rechenschaftspflichtig?). ${ }^{88}$

Wie sich zeigen wird, enthält bereits das Primärrecht basale Qualitätsvorgaben, die auf diese Dimensionen einwirken und die Organe zu legitimationsförderlicher Öffentlichkeitsarbeit anhalten. Hierbei muss nicht übermäßig auf die abstrakten Werte des Art. 2 EUV abgestellt werden, hinsichtlich derer auch der EuGH in seiner bisherigen Praxis grundsätzlich davon abgesehen hat, sie als Maßstab für die Entscheidung von Rechtssachen heranzuziehen. ${ }^{89}$ Aus diesen müssen auch keine - wie es der EuGH richterrechtlich insbesondere hinsichtlich des Rechtsstaatsprinzips getan hat ${ }^{90}$ - substanzangereicherte Rechtsgrundsätze herausgearbeitet werden. Das geschriebene Primärrecht enthält diese inzwischen selbst. Es wurde vor allem durch den Vertrag von Lissabon durch eine Vielzahl konkreterer Vorgaben für die Öffentlichkeitsarbeit erweitert.

86 EuGH, Urteil v. 24.11.1982, C-249/81, ECLI:EU:C:1982:402 - Buy Irish.

87 BVerfGE 89, 155 (185) - Maastricht; BVerfGE 123, 267 (358) - Lissabon.

$88 \operatorname{Meyer}$ (Fn. 2), 622 f.; vgl. demgegenüber das Konzept von Brüggemann (Fn. 2), $89 \mathrm{ff}$.

89 Meinhard Hilf/Frank Schorkopf, in: Grabitz/Hilf/Nettesheim (Fn. 67), Art. 2 EUV Rn. 46.

90 Vgl. Armin von Bogdandy, Tyrannei der Werte, ZaöRV 79 (2019), 503-551 (536); Frank Schorkopf, Der Wertekonstitutionalismus der Europäischen Union, JZ 75 (2020), 477-485 (481); Christian Calliess, in: Christian Calliess/Matthias Ruffert, EUV/AEUV, Art. 2 EUV Rn. 26, jeweils m.w. N. 


\section{Thematikdimension}

\section{a) Pflicht zur Öffentlichkeitsarbeit}

Vor den Änderungen des Primärrechts durch den Vertrag von Lissabon waren zwar bereits Transparenzvorgaben etabliert, proaktive Öffentlichkeitsarbeit war dagegen weder als Kompetenz noch als Pflicht ausdrücklich normiert. Entsprechende Handlungen der EU-Organe fanden im rechtlichen Graubereich statt.

Wollte man eine Kompetenz konstruieren, wäre dies auf zwei Wegen denkbar gewesen. Zum einen hätte Öffentlichkeitsarbeit als implied power, als eine durch den EuGH anerkannte ${ }^{91}$ implizite Kompetenz kraft Sachzusammenhangs verstanden werden können. Als einen solchen Annex zur jeweiligen Sachkompetenz folgert der EuGH auch etwa die Sanktionsmöglichkeit, um eine wirksame Durchsetzung von unionsrechtlichen Vorgaben zu ermöglichen. ${ }^{92}$ Deutschland hätte insoweit als Vorbild dienen können, als auch im dortigen Verfassungsrecht Annexkompetenzen zu geschriebenen Kompetenzen hinzutreten können um mit der Materie zusammenhängende Vorbereitungs- und Durchführungsstadien miteinzubeziehen. ${ }^{93}$ In der Folge wird dort gerade die Kompetenz zur Öffentlichkeitsarbeit meist als solcher Annex zur eigentlichen Sachaufgabe verstanden. ${ }^{94}$ Als alternativer Anknüpfungspunkt wäre die Flexibilitätsklausel des Art. 352 Abs. 1 AEUV in Betracht gekommen, ${ }^{95}$ die nach verbreiteter Ansicht trotz des Begriffs der „Vorschrift" in der deutschen Sprachfassung nicht auf rechtsförmige Handlungen

91 Vgl. EuGH, Urteil v. 29.11.1956, Rs. 8/55, Slg 1955-56, 297, 312 - Belgien/Hohe Bebörde; EuGH, Urteil v. 15.7.1960, Rs. 20/59, Slg 1960, ECLI:EU:C:1960:33, 681, 708 - Italien/Hohe Behörde; EuGH, Urteil v. 15.7.1960, Rs.25/59, Slg 1960, ECLI:EU:C:1960:34, 743, 781 Niederlande/Hohe Behörde; EuGH, Urteil v. 27.9.1988, Rs.165/87, Slg 1988, ECLI:EU: C:1988:458, 5545, Rn. 8 - Kommission/Rat; Stefan Kadelbach, in: von der Groeben/Schwarze/ Hatje (Fn. 67), Art. 5 EUV Rn. 11.

92 EuGH, Urteil v. 27.10.1992, Rs. C-240/90, ECLI:EU:C:1992:408, Rn. 11 ff. - Deutschland/Kommission.

93 Jochen Rozek, in: Hermann von Mangoldt/Friedrich Klein/Christian Starck, GG, 7. Aufl., München: C. H. Beck 2018, Art. 70 Rn. 48; vgl. BVerfGE 88, 203, 331; BVerfGE 109, 190, 215; BVerfGE 113, 348, 369.

94 Christoph Gusy, Informationsbeziehungen zwischen Staat und Bürger, in: Wolfgang Hoffmann-Riem/Eberhard Schmidt-Aßmann/Andreas Voßkuhle (Hrsg.), Grundlagen des Verwaltungsrechts, Bd. 2, München: C.H. Beck 2012, $\mathbb{2 3}$ Rn. 98; Christoph Gusy, Verwaltung durch Information, NJW 53 (2000), 977 (980); Edmund Brandt, Umweltaufklärung und Verfassungsrecht, Taunusstein: Blottner 1994, 124; Hubertus Gersdorf, Staatliche Kommunikationstätigkeit, AfP 4/2016, 293-301 (294); Albert Ingold, „Polizei 2.0“: Grenzen der behördlichen Öffentlichkeitsarbeit in sozialen Netzwerken, VerwArch 108 (2017), 240-265 (248).

95 Hier verortet die Kompetenz Brüggemann (Fn. 2), 128. 
begrenzt ist. ${ }^{96}$ Die zur Kompetenzergänzung erforderliche Verfolgung eines Unionsziels (Art. 3 Abs. 1 EUV) läge für die Öffentlichkeitsarbeit in der Förderung des demokratischen Wertes aus Art. 2 EUV.

Inzwischen stellt jedoch Art. 11 Abs. $2 \mathrm{EUV}$, der zumindest für die Organe im Sinne des Art. 13 Abs. 2 S. 1 EUV einen offenen, transparenten und regelmäßigen Dialog mit der Zivilgesellschaft fordert, die Kompetenz und auch die Pflicht zur Öffentlichkeitsarbeit auf. Da die Vorschrift diesen Dialog in der anderen Alternative auch mit repräsentativen Verbänden fordert, wird systematisch deutlich, dass mit dem Begriff der Zivilgesellschaft ein darüberhinausgehender, unorganisierter Personenkreis gemeint ist. Die ratio legis liegt gerade darin, die Entstehung eines Raumes der europaweiten und EUbezogenen Kommunikation, eine europäische Öffentlichkeit zu fördern. ${ }^{97}$ Die Vorschrift erweitert die vielzähligen Transparenzvorgaben (Art. 1 Abs. 2 EUV, Art. 10 Abs. 3 S. 2 EUV, Art. 15 Abs. 1, 3 UAbs. 3 AEUV) um ein proaktives Element, indem sie selbst zu einem Dialog mit der Zivilgesellschaft als Ganzes aufruft und nicht lediglich die Dokumentenbereitstellung gegenüber interessierten Einzelnen fordert. Die unionsrechtlichen Vorgaben gehen hier weiter, als etwa das deutsche Verfassungsrecht, aus welchem das BVerfG bislang eine Pflicht zur Öffentlichkeitsarbeit lediglich für die Bundesregierung gefolgert hat. ${ }^{98}$

Wortlaut und Systematik des Art. 11 Abs. 2 EUV sprechen gegen eine Pflicht zu einer absoluten, jeden unionalen Vorgang erfassenden Öffentlichkeitsarbeit. Der Dialog mit der Zivilgesellschaft soll „regelmäßig“, nicht ständig stattfinden. ${ }^{99}$ Es wäre auch im Vergleich mit Art. 15 Abs. 1 AEUV, („weitestgehende“ Offenheit) unsinnig, über proaktive Öffentlichkeit eine weitergehende Öffnung zu verlangen, als sie im Sinne reiner Transparenz gefordert wird. Die Abstraktheit der Norm spricht dagegen, ihr Vorgaben für bestimmte Öffentlichkeitsarbeit zu bestimmten Zeitpunkten zu entnehmen. Die EU-Organe haben diesbezüglich einen Gestaltungs- und Ermessensspielraum, sie dürfen im Sinne eines Untermaßverbots aber nicht gänzlich auf

96 Vgl. Rudolf Streinz, in: Rudolf Streinz, EUV/AEUV, 3. Aufl. 2018, Art. 352 AEUV Rn. 45; Daniela Winkler, in: Grabitz/Hilf/Nettesheim (Fn. 67), Art. 352 AEUV Rn. 91.

97 Roland Bieber, in: von der Groeben/Schwarze/Hatje (Fn. 67), Art. 11 EUV Rn. 3; bedauerlicherweise bezieht sich Art. 11 Abs. 1, 2 EUV nicht auf die Hilfseinrichtungen und Agenturen, obwohl diese erheblich kommunizieren, Roland Bieber, in: von der Groeben/ Schwarze/Hatje (Fn. 67), Art. 11 EUV Rn. 9.

98 BVerfGE 44, 125 (147); BVerfGE 63, 230 (242 f.).

99 Auch andere Sprachfassungen lassen sich eher als regulärer, regelmäßiger Dialog deuten: engl. „regular“, frz. „régulier“, esp. „regular“, ita. „regolare“. Eine Pflicht, die Kommunikationsbeziehungen zu den Unionsbürgern zu verfestigen, sehen Sebastian Heselhaus, in: Matthias Pechstein/Carsten Nowak/Ulrich Häde, Frankfurter Kommentar, 1. Aufl. 2017, Art. 11 EUV Rn. 31; PeterM. Huber, in: Streinz (Fn. 96), Art. 11 EUV Rn. 25. 
Öffentlichkeitsarbeit verzichten ${ }^{100}$ und müssen diese als Regelfall anstatt als Ausnahme begreifen.

\section{b) Prinzip der begrenzten Einzelermächtigung}

Mit der Kompetenz und Pflicht zur Öffentlichkeitsarbeit ist noch nichts über die legitimen Gegenstände und Thematiken derselben gesagt. In der Politikwissenschaft wird befürchtet, dass gerade in Bereichen, in denen die Europäische Union wenige oder keine eigenen Kompetenzen hat, ein Anreiz für diese bestehen könnte, Agenda Setting zu betreiben um die eigene Position zu kräftigen. ${ }^{101}$ Ein in der politischen Praxis hierfür standardmäßig vorgebrachtes Argument lautet, es sei vorteilhaft, gemeinsame Probleme der Mitgliedstaaten auch gemeinsam anzugehen. ${ }^{102}$

$\mathrm{Ob}$ aus dem Prinzip der begrenzten Einzelermächtigung (Art. 5 Abs. 1 S. 1 EUV), das für alle Organe der Union gilt (Art. 13 Abs. 1 UAbs. 2 EUV), eine inhaltliche Begrenzung der Öffentlichkeitsarbeit gefolgert werden kann, bedarf einiger Ausführungen. Das Prinzip bezieht sich zunächst auf die rechtsförmigen Handlungsformen des Art. 288 UAbs. 2-4 AEUV. Es ist bereits umstritten, ob unverbindliche Empfehlungen, Stellungnahmen (Art. 288 UAbs. 5 AEUV) und Mitteilungen ${ }^{103}$ unter das Prinzip der begrenzten Einzelermächtigung fallen. Für eine Erstreckung des Prinzips spricht zunächst, dass auch nichtrechtsförmige Handlungen Steuerungswirkung haben können und die Unionsorgane insgesamt zu befugnisgemäßem Handeln aufgerufen sind (Art. 13 Abs. 2 EUV). ${ }^{104}$

100 Vgl. Sebastian Heselhaus, in: M. Pechstein/C. Nowak/U. Häde (Fn. 99), Art. 11 EUV Rn. 31; vgl. zu Art. 11 Abs. 1 EUV Peter M. Huber, in: Streinz (Fn. 96), Art. 11 AEUV, Rn. $9 f$.

101 M. Haverland/M. de Ruiter/S. Van de Walle (Fn. 21), 327 (331); Princen (Fn. 38), 936 ff.; vgl. Meyer (Fn. 8), 1056.

102 Vgl. Princen (Fn. 38), 938.

103 Wohl für eine Geltung des Prinzips Reginhard Henke, Die Leitlinien der Kommission Instrument zur Gewährleistung eines einheitlichen Verwaltungsvollzugs im europäischen Zollrecht?, in: Gerrit Manssen/Monika Jachmann/Christoph Gröpl (Hrsg.), Nach geltendem Verfassungsrecht, Festschrift für U. Steiner, Stuttgart/München: Richard Boorberg 2009, 274 (287 f.); Linda Senden, Soft Law in European Community Law, Oxford: Hart 2004, 312 ff.; a. A. M. Knauff, Der Regelungsverbund: Recht und Soft Law im Mehrebenensystem, Tübingen: Mohr Siebeck 2010, $402 \mathrm{f}$.

104 Stefan Kadelbach, in: von der Groeben/Schwarze/Hatje (Fn. 67), Art. 5 EUV Rn. 12; Martin. Nettesheim, in: Grabitz/Hilf/Nettesheim (Fn. 67), Art. 288 AEUV Rn. 200; a. A. H.-P. Kraußer, Das Prinzip begrenzter Ermächtigung im Gemeinschaftsrecht als Strukturprinzip des EWG-Vertrags, Berlin: Duncker \& Humblot 1991, 88 f.; Bernd Biervert, Der Mißbrauch von Handlungsformen im Gemeinschaftsrecht, Baden-Baden: Nomos 1999, 90. 
Öffentlichkeitsarbeit unterscheidet sich indes noch einmal von diesen - in Art. 288 AEUV nicht abschließend aufgezählten (arg. Art. 296 Abs. 3 AEUV) - Handlungsformen. Mit ihr sind keinerlei rechtlich verbindliche Wirkungen intendiert, sie ist als reines Informationshandeln sämtlichem Unionsrecht nachrangig und auch nicht der untersten tertiären Ebene des Unionsrechts als soft law zuzuordnen. ${ }^{105}$ Öffentlichkeitsarbeit ist eine unverbindliche Handlungsform ohne Rechtscharakter, die nicht in Art. 288 AEUV, auch nicht in dessen UAbs. 5 enthalten ist. Sie ist trotz ihrer Steuerungswirkungen und Gefährdungspotenziale wesentlich schwächer formalisiert als Erklärungen oder Stellungnahmen, die im Amtsblatt der Union veröffentlicht werden können und teilweise Prozessvoraussetzungen vor dem EuGH darstellen. ${ }^{106}$

Man könnte auch deswegen skeptisch sein, ob das Prinzip der begrenzten Einzelermächtigung anzuwenden ist, weil Art. 11 Abs. 2 EUV explizit und themenübergreifend $\mathrm{zu}$ Öffentlichkeitsarbeit ermächtigt (und verpflichtet). Erst aus der Funktion der Öffentlichkeitsarbeit folgt eine mittelbare Koppelung an das Prinzip der begrenzten Einzelermächtigung. Diese liegt gerade darin, die Rahmenbedingungen einer Europäischen Öffentlichkeit zur Stärkung der demokratischen Legitimation zu schaffen. Demokratieförderlich ist Öffentlichkeitsarbeit aber nur dann, wenn sie sich darauf beschränkt, das Mandat der Europäischen Union und ihr entsprechendes Handeln sichtbar $\mathrm{zu}$ machen. In die nationalen Kompetenzräume vorzudringen und dort europäische Mitsprache zu erzwingen, wäre dagegen kontraproduktiv. Öffentlichkeitsarbeit muss daher immer an eine Sachkompetenz der Union gekoppelt sein.

\section{c) Fehlinformation und Manipulation}

Das europäische Primärrecht wirkt nicht nur auf das „Ob“ und das „Was“ der Öffentlichkeitsarbeit ein, sondern auch auf das „Wie“. Insoweit stellen

105 Vgl. Jürgen Schwarze, Soft Law im Recht der Europäischen Union, EuR 46 (2011), 318; Armin von Bogdandy/Jürgen Bast/Felix Arndt, Handlungsformen im Unionsrecht, ZaöRV 62 (2002), 77-161 (120 ff.).

106 Stellungnahmen bilden etwa Prozessvoraussetzungen von Klagen vor dem EuGH, Art. 258, Art. 259 Abs. 3, 4, Art. 265 Abs. 2 AEUV und werden in der Amtsblatt-Serie L veröffentlicht. Zur auslegungsdirektiven Wirkung von Empfehlungen gegenüber nationalen Gerichten EuGH, Urteil v. 13.12.1989, Rs. 322/88, Slg. 1989, 4407, ECLI:EU:C:1989:646 Rn. 18 - Grimaldi; EuGH, Urteilv. 21.1.1993, Rs. C-188/91, Slg. 1993, I-363, ECLI:EU: C:1993:24 Rn. 18 - Deutsche Shell AG; vgl. insgesamt von Bogdandy/ Bast/Arndt (Fn. 105), 77 (116ff.); Martin Nettesheim, in: Grabitz/Hilf/Nettesheim (Fn. 67), Art. 288 AEUV Rn. 206; Werner Schroeder, in: Streinz (Fn. 96), Art. 288 Rn. 32, 132. 
die Rechtstexte keine expliziten Gebote auf, jedoch enthalten sie Vorgaben, die denknotwendig gewisse Fehlpraktiken und Defizite der Öffentlichkeitsarbeit ausschließen.

Zunächst verdeutlicht Art. 2 EUV, dass die Würde des Menschen (Art. 1 $\mathrm{GrC}$ ) an der Spitze der Werte der Europäischen Union steht und die Grundlage für alle ihre Maßnahmen bildet. ${ }^{107}$ Die Menschenwürde mag vage und programmatisch erscheinen, aber das zugrunde liegende Menschenbild schließt zumindest einige Kommunikationsstrategien aus. Ein zentraler Inhalt der Menschenwürde liegt in der Achtung der Selbstbestimmtheit der Menschen, welche es impliziert, ihre Instrumentalisierung und Objektifizierung zu verbieten. ${ }^{108}$ Auf einer Linie hiermit betonte der EuGH in seiner Entscheidung zur Richtlinie über den rechtlichen Schutz biotechnologischer Erfindungen - wenn auch in einem anderen Zusammenhang -, dass der menschliche Körper unverfügbar und unveräußerlich sei und vor durch Drittinteressen motivierten Eingriffen geschützt werde. ${ }^{109}$ Die menschliche Würde zu achten, bedeutet auch, die körperliche und geistige Integrität der Menschen anzuerkennen.

Diese Erwägungen lassen sich mutatis mutandis auf ein der Menschenwürde entsprechendes Rezipientenbild der Öffentlichkeitsarbeit übertragen: A priori ausgeschlossen sind hiernach solche Kommunikationsstrategien, die gerade keinen kritisch-selbstbestimmten Umgang mit sich ermöglichen wollen, sondern in täuschender oder manipulativer Intention erfolgen. Die denkbaren Techniken werden regelmäßig durch kommunikationswissenschaftliche und kognitionspsychologische Forschungsergebnisse fortgeschrieben. Sie können in der übermäßigen Nutzung von Framing- oder Priming-Effekten, ${ }^{110}$ in der subliminalen Beeinflussung durch unterschwellige Reize oder im schlichten Lügen ${ }^{111}$ liegen. Bei diesen Phänomenen zeigt sich letztlich

107 Walter Frenz, in: Pechstein/Nowak/Häde (Fn. 99), Art. 1 GRC Rn. 18.

108 Vgl. Matthias Mablmann, Grundrechtstheorien in Europa - kulturelle Bestimmtheit und universeller Gehalt, EuR 46 (2011), 469-487 (480) m. w. N.

$109 \mathrm{EuGH}$, Urteil v. 9.10.2001, Rs. C-377/98, Slg. 2001, I-7079, ECLI:EU:C:2001:523, Rn. 77 - Niederlande/Parlament und Rat.

110 Vgl. Robert M. Entman, Toward Clarification of a Fractured Paradigm, Journal of Communication 43 (1993), 51-58; Robert M. Entman, Cascading Activation: Contesting the White House's Frame After 9/11, Political Communication 20 (2003), 415-432 (417); Jörg Matthes, Framing, Baden-Baden: Nomos 2014, 10 ff.; Jochen Peter, Medien-Priming - Grundlagen, Befunde und Forschungstendenzen, Publizistik 47 (2002), 21-44; Dirk Wentura/Klaus Rothermund, Priming is not Priming is not Priming, Social Cognition 32 (2014), 47-67.

111 Vgl. Jean-Nicolas Druey, Liability for Deficient Statements - An Approach under Information Law, in: Urs Gasser (Hrsg.), Information Quality Regulation, Zürich: Schulthess 2004, 189: „Non-information, in the sense of the failure to give information where the law would so require, is just another case of bad information, that is to say of quality deficiency. We can observe that, indeed, in both cases the value of information is missing for the recipient. Bad 
auch der Zusammenhang von Menschenwürde und Demokratieprinzip: Der demokratische Grundgedanke einer Selbstherrschaft der Unionsbürger wäre gerade deswegen konterkariert, weil deren informationellen und kognitiven Bedingungen zugunsten einer Instrumentalisierung des Einzelnen manipuliert würden.

Gestützt wird diese Erkenntnis durch die Verwendung des Begriffs Dialog in Art.11 Abs. 2 EUV, der ebenfalls einige Qualitätsvorgaben an die Öffentlichkeitsarbeit impliziert. Bereits Platon prägte den Begriff als auf intellektuellen Argumentationsaustausch ausgerichtet und noch heute wird der Begriff Kommunikationsform normativ aufgeladen. ${ }^{112}$ Nach tradiertem Verständnis findet ein Dialog auf Augenhöhe statt und versucht Informationsasymmetrien im Laufe des Gesprächs abzubauen. Er ist auf Verständigung mit dem Gegenüber ausgerichtet, wofür die eigene Position möglichst klar präsentiert werden muss. In diesem Zusammenhang ist auch Art. 11 Abs. 2 GrC aufschlussreich, der das Institut freier, pluraler Medien statuiert und diesen die Funktion des public watchdog gegenüber der Europäischen Union überantwortet. Mit dieser Gewährleistung wäre es nicht zu vereinbaren, wenn die Union als herausgehobenes Beobachtungsobjekt diese Funktion doch wieder unterwanderte, indem sie über ihre Öffentlichkeitsarbeit eine maßgebliche mediale Informationsgrundlage für ihre Beobachtung und Vermittlung sabotierte.

Neben die genannten Vorgaben tritt hier das Transparenzgebot als Leitmotiv der Neufassung des Primärrechts durch den Vertrag von Lissabon. ${ }^{113}$ Insbesondere das in diesem Zusammenhang geschaffene Grundrecht auf Zugang zu Dokumenten (Art. $42 \mathrm{GrC}$ und Art. 15 Abs. 3 AEUV) und die konkretisierende Transparenz-VO (VO 1049/2001/EG) sind aus demokratischen Erwägungen vom Grundsatz des größtmöglichen Zugangs der Öffentlichkeit zu Dokumenten getragen. ${ }^{114}$ Nun könnte man argumentieren, dass

information - and herein also lies the difference from a bad car - is not by itself more than noninformation. It cannot derive any value from being information after all, i.e. from constituting at least a step in the good direction. Bad information, for example misleading information, may even have a negative value, because it induces a detrimental behaviour on the part of the recipient." (Hervorhebungen im Original).

112 Vgl. Katarzyna Jazdzewska, From Dialogos to Dialogue: The Use of the Term from Plato to the Second Century CE, Greek, Roman, and Byzantine Studies 54 (2014), 17-36 (23 f.); Louise Phillips, The Promise of Dialogue, Amsterdam: John Benjamins Publishing 2011, $1 \mathrm{ff}$.

113 Thomas von Danwitz, Öffentlichkeit, Transparenz und Vermittlung von Rationalität in der Rechtsprechung des Gerichtshofes der Europäischen Gemeinschaften, in: Pernice/Otto (Fn. 34), 29 (31).

114 Vgl. Erwägungsgrund 2 und 4 sowie EuGH Rs. C-175/18 P, ECLI:EU:C:2020:23, Rn. 56 - PTC Therapeutics International/Europäische Arzneimittelagentur; zu aktuellen Entwicklungen Jens Brauneck, Kein Zugang zu Dokumenten: Politischer Handlungsspielraum der EU-Kommission in Gefahr?, NVwZ 35 (2016), 489-496. 
das Recht auf möglichst weitgehenden Dokumentenzugang gerade in ein Komplementärverhältnis zu einer eklektischen und ermessensgeleiteten Öffentlichkeitsarbeit trete. ${ }^{115}$ Wesentlicher erscheint aber der Systembruch, der zwischen einer möglichst weitgehenden reaktiven Transparenz und einer propagandistischen Öffentlichkeitsarbeit bestünde. Dem Transparenzgebot wohnt der Gedanke inne, die Distanz zwischen Bürgern und Entscheidungsträgern zu überbrücken. ${ }^{116}$ Wenn aber wirklich Distanz überbrückt werden soll, genügt keine Schein-Nähe durch einen Pseudo-Dialog mit verdeckten Karten.

Aus dem Gesagten lassen sich auch Richtigkeitsvorgaben an die Öffentlichkeitsarbeit der Europäischen Union ableiten. Es entspräche sicherlich in dem einen oder anderen Fall der Unionsräson, durch Fehlinformationen die eigene Position zu wahren oder zu stärken - sei es, indem eigene Mängel geleugnet oder Praktiken Dritter falsch dargestellt werden. Einem solchen Vorgehen schiebt das Primärrecht indes einen Riegel vor. Zwar erwartete man zu viel von der Leistungsfähigkeit des Rechts, wollte man auf rechtlichem Wege die EU-Organe dazu bewegen, stets eine an objektiver Wahrheit ausgerichtete Öffentlichkeitsarbeit zu betreiben. So ein Richtigkeitsbegriff lässt sich von der Warte subjektiv-konstruktivistischer und systemtheoretischer Informationsverständnisse als unbrauchbarer Maßstab kritisieren. ${ }^{117} \mathrm{Er}$ wäre auch deswegen impraktikabel, weil sich EU-Organe teils während laufender Erkenntnisprozesse unter Restunsicherheiten äußern und den Unionsbürgern gerade auch die lautere Selbsteinschätzung und Erwartungskundgabe wichtig sein dürfte. Eine an Transparenz und Dialog orientierte Öffentlichkeitsarbeit ist jedoch stets um subjektive Richtigkeit bemüht und verbreitet nicht bewusst Unwahrheiten.

115 Vgl. Marion Albers, Grundlagen und Ausgestaltung der Informationsfreiheitsgesetze, Zeitschrift für das Juristische Studium 6/2009, 614-624 (619); Anke Engelbert/Martin Kutscha, Staatliche Öffentlichkeitsarbeit und Demokratieprinzip, NJW 46 (1993), 1233-1237 (1235f.); Arno Scherzberg, Öffentlichkeitskontrolle, in: Wolfgang Hoffmann-Riem/Eberhard SchmidtAßmann/Andreas Voßkuhle (Hrsg.), Grundlagen des Verwaltungsrechts Bd. 3, München: C. H. Beck 2013, $₫ 49$ Rn. 16.

116 Martin Nettesheim, in: Grabitz/Hilf/Nettesheim (Fn. 67), Art. 1 EUV Rn. 32; Art. 10 EUV Rn. 91.

117 Vgl. Peter L. Berger/Thomas Luckmann, The Social Construction of Reality, New York: Anchor 1967; Eberhard Schockenhoff, Zur Lüge verdammt?, 2. Aufl., Freiburg i. Br.: Herder Verlag 2005, $298 \mathrm{ff}$;; aus der Rechtswissenschaft Wolfang Hoffmann-Riem, Methoden einer anwendungsorientierten Verwaltungsrechtswissenschaft, in: Eberhard Schmidt-Aßmann/Wolfgang Hoffmann-Riem (Hrsg.), Methoden der Verwaltungsrechtswissenschaft, Baden-Baden: Nomos 2004, 30 f.; Arno Scherzberg, Wissen, Nichtwissen und Ungewissheit im Recht, in: Christoph Engel/Jost Halfmann/Martin Schulte (Hrsg.), Wissen - Nichtwissen - Unsicheres Wissen, Baden-Baden: Nomos 2002, $114 \mathrm{ff}$. 


\section{d) Unverständliches und Verkürzungen}

Hinsichtlich zweier verwandter Qualitätsdimensionen, nämlich der Klarbeit und Vollständigkeit der Öffentlichkeitsarbeit, agiert die Europäische Union unter erschwerten Bedingungen, die den Nationalstaaten nicht vergleichbar sind. Öffentliche Institutionen orientieren sich mit ihrer Öffentlichkeitsarbeit regelmäßig an der Allgemeinheit und verfolgen aus demokratischen Erwägungen einen inklusiven Ansatz. ${ }^{118}$ Die Europäische Union sieht sich hierbei aber 450 Millionen Unionsbürgern gegenüber, die unterschiedliche Sprachen sprechen ${ }^{119}$ (Europarechtler kennen das Problem der unterschiedlichen Sprachfassungen des Primär- und Sekundärrechts) und bei der Rezeption unterschiedliche national-historische Erfahrungen und Deutungsmuster anlegen. Zwar können sich Unionsbürger am politischen Meinungsbildungs- und Entscheidungsprozess nur wirksam beteiligen, wenn sie die Thematiken und ihre Zusammenhänge tatsächlich verstehen. Ein intersubjektives Verständnis der Öffentlichkeitsarbeit ist unter diesen Bedingungen aber unwahrscheinlich. Aus der Sprachwissenschaft wissen wir, dass Klarheit keine objektive Eigenschaft eines Kommunikats ist, sondern stets individuell, sozial und historisch auf der Basis von Vorkenntnissen, Erwartungen und Interessen bestimmt werden muss. ${ }^{120}$ Dem nahe stehend stellt Vollständigkeit im Sinne der hinreichenden Kontextualisierung des Gesagten eine Wertung dar, die stets den konkreten Wissensbestand der Rezipienten mitberücksichtigen muss. ${ }^{121}$

Dieser Befund mag ernüchternd sein, dennoch versucht das Primärrecht dem etwas entgegenzusetzen: Die zahlreichen Sprachenrechte in Art. 20 Abs. 2 UAbs. 1 S. 2 lit. d, Art. 24 Abs. 4 AEUV und Art. 41 Abs. 4 GrC, die als „Brückenrechte“ und „Gelingensvoraussetzung europäischer Demokratie " bezeichnet werden, ${ }^{122}$ verdeutlichen den inklusiven, auf tatsächliche Verständigung ausgerichteten Ansatz der Europäischen Union. Dass nach Art. 1

118 Brüggemann (Fn. 2), 289.

$119 \mathrm{Vgl}$. Hermann-J. Blanke/Stefan Pilz, Europa 2019 bis 2024 - Wohin trägt uns der Stier? - Sieben Thesen zu den Herausforderungen der Europäischen Union -, EuR 55 (2020), 270-301 (297).

120 Markus Nussbaumer, Von Schwärmern und Skeptikern und ein Versuch, Realist zu sein. Bilanz und Entwurf des Sprachspiels vom unverständlichen Gesetz, in: Kent D. Lerch (Hrsg.), Die Sprache des Rechts, Bd. 1, Berlin: de Gruyter 2004, 285-295 (289); Kent D. Lerch, Ultra posse nemo obligatur. Von der Verständlichkeit und ihren Grenzen, in: Karin M. EichhoffCyrus (Hrsg.), Verständlichkeit als Bürgerrecht?, Mannheim/Leipzig/Wien/Zürich: Dudenverlag 2008, 54-80 (74); Helmut Ebert, Verständlichkeit und Vertrauen - Die kommunikative Seite der Rechts- und Verwaltungssprache, in: Hans-R. Fluck/Michaela Blaha (Hrsg.), Amtsdeutsch a. D.?, 45-58 (47).

121 Mast (Fn. 71), $278 \mathrm{ff}$.

122 Jörg P. Terbechte, in: Pechstein/Nowak/Häde (Fn. 99), Art. 41 GRC Rn. 21. 
Abs. 2, Art. 10 Abs. 3 S. 2 EUV Entscheidungen möglichst offen und möglichst bürgernah getroffen werden müssen, spricht für eine laienverständliche Sprache und im besten Falle für zielgruppenorientierte Formulierung. ${ }^{123}$ Der Begriff der „Entscheidungen“ ist hierbei im weitest möglichen Sinne zu verstehen; er umfasst nicht lediglich rechtlich bindende Maßnahmen, sondern die gesamte Organisation des politischen Prozesses. ${ }^{124}$ Schließlich deutet der systematisch im Titel II (Bestimmungen über die demokratischen Grundsätze) verankerte Art. 9 S. 1 EUV in diese Richtung, wenn er bestimmt, dass allen Unionsbürgern ein gleiches Maß an Aufmerksamkeit seitens der Organe, Einrichtungen und sonstigen Stellen der Union zuteilwird. Die Vorgabe scheint auf den ersten Blick nicht recht zur demokratischen Willensbildung $\mathrm{zu}$ passen, die primär bei den Unionsbürgern, nicht in den EU-Organen stattzufinden hat. ${ }^{125}$ Sinnvoll wird sie erst, wenn man sie im Zusammenhang mit Art. 11 EUV, insbesondere der darin verankerten Pflicht zur Öffentlichkeitsarbeit begreift: Gleiche Aufmerksamkeit meint hier eine alle Sprachen und Kulturräume berücksichtigende Informationspolitik. Vereinzelt wird hieraus auch das Verbot gefolgert, europakritische Positionen im Diskurs übermäßig zu stigmatisieren. ${ }^{126}$ Die Europäische Union ist deswegen dazu angehalten, sich um tatsächliche Verständigung mit dem jeweiligen Rezipientenkreis zu bemühen und keine Elemente zu verschweigen, die für diese wesentlich sind. Dies liegt auf einer Linie mit der schon vor Jahren von Wallström geforderten Kommunikationsstrategie, jedes Publikum in der für es verständlichen Sprache und auf den von ihm genutzten Kanälen anzusprechen. ${ }^{127}$

So wichtig Klarheits- und Vollständigkeitsvorgaben sein mögen, stellen sie kein Allheilmittel für die Defizite europäischer Öffentlichkeitsarbeit dar. So darf einfache Sprache etwa nicht mit übermäßiger Komplexitätsreduktion ${ }^{128}$ einhergehen, die den Sinn des Geäußerten verzerrt. Die EU-Ebene zeichnet sich durch komplexe Entscheidungsfindungsprozesse aus, deren Darstellung viel Platz erfordert ${ }^{129}$ und die nicht einfach bis zur Verfälschung vereinfacht werden dürfen. Gerade für solche Kommunikationsstrategien besteht seitens der Europäischen Union aber ein erheblicher Anreiz, denn je einfacher ein Thema zu verpacken und in attraktiver Weise einem breiteren Publikum zu

123 Vgl. Marcel Haag, in: von der Groeben/Schwarze/Hatje (Fn. 67), Art. 10 EUV, Rn. 18.

124 Martin Nettesheim, in: Grabitz/Hilf/Nettesheim (Fn. 67), 69. EL 2020, Art. 1 EUV Rn. 33.

125 Sebastian Heselhaus, in: Pechstein/Nowak/Häde (Fn. 99), Art. 9 EUV Rn. 19.

126 Sebastian Heselhaus, in: Pechstein/Nowak/Häde (Fn. 99), Art. 11 EUV Rn. 16.

127 Wallström (Fn. 73), 25.

128 Diese wird aus kommunikationswissenschaftlicher Perspektive von der Europäischen Union gefordert, vgl. Brüggemann (Fn. 2), $32 \mathrm{f}$.

129 Meyer (Fn. 2), 631. 
präsentieren ist, desto eher werden Journalisten solche Transformationsbemühungen auch tatsächlich in Angriff nehmen. ${ }^{130}$ Hier zeigt sich ein Spannungsfeld mit den Richtigkeitsvorgaben. Auch wenn dezentralisiert eine zielgruppenorientierte Öffentlichkeitsarbeit erfolgen soll, dürfen sich die in verschiedenen Mitgliedstaaten kommunizierten Inhalte nicht widersprechen. ${ }^{131}$

Eine Pflicht zur ausgewogenen Darstellung auch gegen die eigene Position sprechender Belange wird man der Öffentlichkeitsarbeit dagegen wohl nicht abverlangen können. Es widerspräche den Logiken des politischen Wettbewerbs, dürften die EU-Organe ihre verfolgten Ziele nicht klar artikulieren. Die Ausgewogenheit wird erst durch das Zusammenspiel der involvierten Akteure hergestellt. Auf einer Linie hiermit liegt die Rechtsprechung des EuGH zur Begründung von Rechtsakten. Hier lässt er es genügen, wenn die erlassende Stelle ihre eigene Auffassung darlegt; sie muss nicht auch andere Ansichten oder Einwände, die im Erlassverfahren aufgekommen sind, thematisieren. ${ }^{132}$

\section{Verfahrensdimension}

Das Primärrecht verspricht für die Verfahrensdimension mehr, als das Sekundärrecht und die Realität einhalten. Nach Ansicht des BVerfG hängt die demokratische Legitimation der Europäischen Union davon ab, ob „die Entscheidungsverfahren der Hoheitsgewalt ausübenden Organe und die jeweils verfolgten politischen Zielvorstellungen allgemein sichtbar und verstehbar sind“. ${ }^{133}$ Gerade diese Qualitätsdimension bespielt die Europäische Union nicht optimal. Wie oben dargelegt, wird empirisch kritisiert, dass insbesondere die Informationstätigkeit der Kommission häufig als nicht direkt mit dem politischen Entscheidungsprozess gekoppelter „Pseudo-Dialog“ organisiert sei. ${ }^{134}$

Dabei enthält das Primärrecht durchaus einige Anhaltspunkte für eine Kommunikationsbeziehung mit den Unionsbürgern bereit, die etwa auch wesentliche Zwischenentscheidungen miteinbezieht: Nach Art. 10 Abs. 3 S. 1 EUV haben die Unionsbürger das Recht, am demokratischen Leben der Union teilzunebmen. Teilnahme meint nach verbreiteter Ansicht nicht allein

$130 \mathrm{Vgl}$. Beyers/Kerremans (Fn. 37), 274.

131 Vgl. Brüggemann (Fn. 2), 104.

132 EuGH, Urteil v. 11.7.1989, Rs. 246/86, Slg 1989, 2117, ECLI:EU:C:1989:301, Rn. 55 Belasco u.a./Kommission; Maria Geismann, in: von der Groeben/Schwarze/Hatje (Fn. 67), Art. 296 AEUV, Rn. 13.

133 BVerfGE 89, 155 (185) - Maastricht.

134 Anm. 51. 
die förmlichen Beteiligungsrechte im Rahmen der Parlamentswahl in Sinne des status activus nach Georg Jellinek, sondern die umfassende kommunikative Partizipation am öffentlichen Meinungsbildungs- und Entscheidungsprozess. ${ }^{135}$ An diesem Prozess teilzunehmen erfordert aber eine Synchronität der politischen Akteure und der Bürger - das Handeln ersterer muss mit dem Wissensbestand letzterer zeitlich abgestimmt sein. Auch dass Art. 11 Abs. 2 EUV einen „regelmäßigen“ Dialog fordert, spricht trotz der oben dargelegten Einschränkungen zumindest für eine zeitliche Nähe. Schließlich wird aus der Anforderung der „Offenheit" des zivilgesellschaftlichen Dialogs in Art. 11 Abs. 2 EUV die Bereitschaft der EU-Organe gefordert, die Argumente der Gegenseite in den noch nicht abgeschlossenen Entscheidungsprozess einfließen zu lassen. ${ }^{136}$

Der EuGH und das Gericht der Europäischen Union $(\mathrm{EuG})$ interpretieren den unionalen Transparenzgrundsatz ähnlich prozedural-partizipativ: „Der Transparenzgrundsatz soll für eine stärkere Teilnahme der Bürger an Entscheidungsprozessen sorgen und eine größere Legitimität, Effizienz und Verantwortung der Verwaltung gegenüber den Bürgern in einem demokratischen System gewährleisten. "137 Hierzu passt, dass nach den Art. 1 Abs. 2 EUV, Art. 10 Abs. 3 S. 2 EUV, Art. 15 Abs. 1, 3 UAbs. 3 AEUV Entscheidungen möglichst offen und möglichst bürgernah getroffen werden müssen. Den Maximen der Offenheit und Bürgernähe wird eine prozedurale Dimension zugeschrieben, wonach auch der Willensbildungsprozess im Vorfeld einer Entscheidung nachvollziehbar sein muss. ${ }^{138}$ Bürgernähe lässt sich in einem prozeduralen Sinne als Einbeziehung der Unionsbürger in den Entscheidungsfindungsprozess deuten. ${ }^{139}$

Trotz dieser vollmundigen Worte delegiert das Primärrecht Einschränkungsregelungen des Dokumentenzugangs an den Verordnungsgeber

135 Marcel Haag, in: von der Groeben/Schwarze/Hatje (Fn. 67), Art. 10 EUV Rn. 11; Peter M. Huber, in: Streinz (Fn. 96), Art. 10 EUV Rn. 47 f.; Sebastian Heselhaus, in: Pechstein/ Nowak/Häde (Fn. 99), Art. 10 EUV Rn. 30; enger Matthias Ruffert, in: Calliess/Ruffert (Fn. 90), Art. 10 EUV Rn. 12.

136 Sebastian Heselhaus, in: Pechstein/Nowak/Häde (Fn. 99), Art. 11 EUV Rn. 33; Peter M. Huber, in: Streinz (Fn. 96), Art. 11 EUV Rn. 21.

137 EuG, Urteil v. 7.2.2002, Rs. T-211/00, ECLI:EU:T:2002:30, Slg. 2002, II-485 Rn. 52 Kuijer/Kommission; EuGH, Urteil v. 9.11.2010, verb. Rs. C-92/09 u. C-93/09, ECLI:EU: C:2010:662, Rn. 68 - Schecke GbR und Eifert/Hessen; vgl. zur TransparenzVO: EuGH, Urteil v. 29.6.2010, Rs. C-28/08 P, ECLI:EU:C:2010:378 - Bavarian Lager Co. Ltd./Kommission; EuGH, Urteil v. 18.12.2007, Rs. C-64/05 P, ECLI:EU:C:2007:802, Rn. 54 - Sweden/Kommission.

138 Martin Nettesheim, in: Grabitz/Hilf/Nettesheim (Fn. 67), Art. 10 EUV Rn. 93; vgl. Franzius/Preuß (Fn. 26) 23 f., $151 \mathrm{f}$.

139 Sebastian Heselhaus, in: Pechstein/Nowak/Häde (Fn. 99), Art. 10 EUV Rn. 39, 41; Martin Nettesheim, in: Grabitz/Hilf/Nettesheim (Fn. 67), Art. 1 EUV Rn. 31. 
(Art. 15 Abs. 3 UAbs. 2 AEUV in Verbindung mit VO 1049/2001/EG Transparenz-VO) und an Sonderbestimmungen in Geschäftsordnungen der EU-Organe (Art. 15 Abs. 3 UAbs. 3 AEUV). Vor unbegrenztem Dokumentenzugang soll Art. 4 Abs. 3 Transparenz-VO gerade den intraorganisatorischen Entscheidungsprozess als Raum freien Denkens schützen und - wenn auch unter gesteigerten Rechtfertigungserfordernissen ${ }^{140}$ auch über den Zeitpunkt der einzelnen Entscheidung hinausgehend geheim halten. Öffentlichkeit und Geheimhaltung so abzuschichten ist indes keine Spezialität der Europäischen Union, sondern auch auf nationaler Ebene verbreitet. Es entspricht einem tradierten politischen Verständnis insbesondere der Gubernative. Auch mit den durch das BVerfG vorgegebenen Transparenzausnahmen liegen sie auf einer Linie. Absolut kann die von dem BVerfG im MaastrichtUrteil geforderte Sichtbarkeit daher nicht gemeint sein, denn das Gericht selbst erkennt für die deutsche Bundesregierung einen nicht ausforschbaren Initiativ-, Beratungs- und Handlungsbereich an, welcher der parlamentarischen Kontrolle entzogen ist (Kernbereich exekutiver Eigenverantwortung). ${ }^{141}$

Zwar fehlen ebensolche Schrankenregelungen und Delegationen für die Öffentlichkeitsarbeit der Europäischen Union. Die im systematischen Vergleich zu den Transparenz- und Dokumentenzugangsregelungen vage gehaltenen Bestimmungen vermögen dennoch keine weitergehende Öffentlichkeit zu erzwingen. All die beschriebenen Artikel konkretisieren, wie die Öffentlichkeitsarbeit als eigenständige, primärrechtlich vorgegebene Aufgabe der EU-Organe betrieben werden muss. Sie stehen allerdings normhierarchisch auf einer Stufe mit den die EU-Organe, ihre Organisation und Verfahren institutionalisierenden Artikeln des Primärrechts. Die Transparenz- und Dialognormen können damit einen Modus der Aufgabenerledigung vorgeben, der die institutionellen und organisatorischen Rahmenvorgaben ausfüllt, aber nicht überlagert. Wie sich aus einem Umkehrschluss aus Art. 15 Abs. 2 AEUV ergibt, beraten die EU-Organe nicht generell öffentlich, sondern lediglich das Europäische Parlament und der Rat (nur) bezüglich Gesetzgebungsakten - daran vermag auch die Pflicht zur Öffentlichkeitsarbeit nach geltender Unionsrechtslage nichts zu ändern.

140 Vgl. zu den mit Abschluss eines Verfahrens gesteigerten Anforderungen an die Geheimhaltungsbegründung EuG, Rs. T-403/05, ECLI:EU:T:2008:316 Rn. 78 - Schweden/MyTravel und Kommission; EuG, Rs. T-111/00, Slg. 2001, II-2997 Rn. 36 ff. - BAT/Kommission; Bernhard W. Wegener, in: Calliess/Ruffert (Fn. 90), Art. 15 AEUV Rn. 32; Sebastian Heselhaus, in: Pechstein/Nowak/Häde (Fn. 99), Art. 15 AEUV Rn. 67; Bernhard Hofstötter, in: von der Groeben/Schwarze/Hatje (Fn. 67), Art. 15 AEUV Rn. 57.

141 BVerfGE 67, 100 (139); BVerfGE 110, 199 (214); BVerfGE 124, 78 (120ff.); BVerfGE 147, 50 (138 ff. Rn. 227 ff.). 
So bleibt der Zeitpunkt der Öffentlichkeitsarbeit noch weitgehend eine politische Ermessensentscheidung. Die Unionsorgane sind nicht verpflichtet, jeden Zwischendisput und jede fallengelassene Forderung simultan oder nachträglich proaktiv zu verbreiten. Auch insoweit vermag der geltenden Unionsrechtslage lediglich ein Untermaßverbot entnommen zu werden: Die Öffentlichkeitsarbeit der EU darf sich nicht völlig vom Entscheidungsprozess abkoppeln.

\section{Verantwortlichkeitsdimension}

Die dritte Qualitätsdimension fordert von der Öffentlichkeitsarbeit, politische Standpunkte oder Handlungen aufzuzeigen und bestimmten Personen oder Institutionen zuzuordnen. Dass dies an Grenzen stößt, wo Entscheidungen innerhalb von Kollegialorganen und Gremien ausgehandelt werden, wurde soeben anhand des Rates dargestellt. In der Regel muss sich die Öffentlichkeit hier damit begnügen, das dem EU-Organ als solchem zurechenbare Endresultat mitgeteilt zu bekommen. Allerdings können auch interorganisatorisch Verantwortlichkeiten verschwimmen. Für dieses Phänomen hält das Primärrecht einige Vorgaben bereit.

Am bedeutsamsten ist hier das Demokratieprinzip, insbesondere in seiner Ausprägung des Transparenzgebots. ${ }^{142}$ Der EuGH erkennt die demokratische Funktion des Transparenzgebots an und betont die hierdurch gesteigerte Legitimität, Effizienz und Verantwortung gegenüber den Unionsbürgern. ${ }^{143}$ Diese allgemeine Organisations- und Handlungsmaxime der Europäischen Union ${ }^{144}$ wird durch Art. 15 Abs. 1 AEUV konkretisiert, indem er die Organe, Einrichtungen und sonstigen Stellen der Union ausdrücklich als Verpflichtungsadressaten benennt. Für die Öffentlichkeitsarbeit der Europäischen Union als einem wichtigen Faktor und Bedingung des europäischen Diskurses, wird das Transparenzgebot in spezifischer Weise relevant: Weil die Funktion der Öffentlichkeitsarbeit nicht gleichermaßen durch andere Akteu-

142 Vgl. EuGH, Urteil v. 30.4.1996, Rs. C-58/94, Slg. 1996 I-02169, ECLI:EU:C:1996:171, Rn. $34 \mathrm{ff}$ - Niederlande/Rat.

$143 \mathrm{EuGH}$, Urteil v. 21.9.2010, verb. Rs. C-514/07 P u.a., Slg. 2010, I-8533 Rn. 68 Schweden u. a./API und Kommission; EuGH, Urteil v. 1.7.2008, verb. Rs. C-39/05 P und C-52/ 05 P, Slg. 2008, I-4723 Rn. 34 - Schweden und Turco/Rat; vgl. Martin Nettesheim, in: Grabitz/ Hilf/Nettesheim (Fn. 67), 69. EL 2020, Art. 1 EUV Rn. 35; Sacha Prechal/Magdalena E. de Leeuw, Transparency: A General Principle of EU Law?, in: Ulf Bernitz/Joakim Nergelius/ Cecilia Cardner (Hrsg.), General Principles of EC Law in A Process of Development, Alphen aan den Rijn: Kluwer Law International 2008, 201-242 (205).

144 Martin Nettesheim, in: Grabitz/Hilf/Nettesheim (Fn. 67), Art. 1 EUV Rn. 27, 34; Bernhard Hofstötter, in: von der Groeben/Schwarze/Hatje (Fn. 67), Art. 15 AEUV Rn. 2. 
re wahrgenommen werden kann und sie spezifischen Qualitätsvorgaben unterliegt, ist es wichtig, dass sie durch die Rezipienten als solche erkannt wird. Träte die Europäische Union als eigentliche Urheberin einer Äußerung zurück und verschleierte ihre Selbst- durch scheinbare Fremddarstellung, könnten die Unionsbürger die verfolgten Kommunikationsziele nicht mehr nachvollziehen, ein selbstbestimmter und kritischer Umgang würde erschwert. ${ }^{145}$ Es muss deswegen stets erkennbar sein, dass die Europäische Union und welches konkrete EU-Organ kommuniziert (Kommunikatorklarbeit $)^{146}$. Zu Unklarheiten kann es insbesondere kommen, wenn EU-Organe untereinander oder mit Drittinstitutionen kollaborieren und wenn sie Gestaltungslogiken anderer Bereiche adaptieren, etwa wenn sie ihre Social MediaAuftritte ähnlich entwerfen wie journalistisch-redaktionelle oder Unterhaltungsangebote. Angesichts des empirisch deutlichen Übergewichts der Öffentlichkeitsarbeit der Kommission ist es für ein adäquates Verständnis der Europapolitik wichtig, sie nicht mit der Europäischen Union als solcher gleichzusetzen.

In gewisser Weise entgegengesetzt hierzu wirkt die Pflicht der EU-Organe zu loyaler Zusammenarbeit untereinander (Art. 13 Abs. 2 S. 2 EUV) und gegenüber den Mitgliedstaaten (Art. 4 Abs. 3 EUV). ${ }^{147}$ Die Normen könnten als primärrechtlicher Anknüpfungspunkt für die oben genannte Interinstitutionelle Vereinbarung Partnerschaft für die Kommunikation über Europa (COM(2007) 569 final) dienen. Ihr Wortlaut spricht dafür, dass sie auch auf die Öffentlichkeitsarbeit anwendbar sind, denn diese zählt, wie wir gesehen haben, aufgrund Art. 11 Abs. 2 EUV zu den Aufgaben, die sich aus den Verträgen ergeben. Auch die ratio legis scheint passend, weil Öffentlichkeitsarbeit durchaus die Ziele und Handlungen anderer politischer Akteure desavourieren und damit erschweren kann. Die normative Kraft der Loyalitätsvorgaben ist allerdings fraglich. Die Europäische Union ist aufgrund ihres institutionellen Zuschnitts „polyphonic by nature“. ${ }^{148}$ Dementsprechend beobachtet die Kommunikationswissenschaft empirisch, dass sämtliche EUOrgane ihre Öffentlichkeitsarbeit nach wie vor autonom betreiben und eine europäische Informationspolitik entlang der Institutionengrenzen fragmen-

145 Vgl. Walter Leisner, Der unsichtbare Staat, Berlin: Duncker \& Humblot 1994, 52; Mast (Fn. 71), 333.

146 Mast (Fn. 71), $336 \mathrm{ff}$.

147 Schon vor den Regelungen betonte der EuGH, dass das institutionelle Gleichgewicht zwischen den Organen der Europäischen Union zu wahren sei und die Organe ihre Befugnisse unter Beachtung der Befugnisse der anderen Organe auszuüben hätten, EuGH, Urteil v. 4.10. 1991, Rs. C-70/88, Slg. 1990, I-2041, ECLI:EU:C:1991:373 Rn. 21 f. - Tschernobyl I; vgl. EuGH, Urteil v. 16.7.1992, Rs. C-65/93, Slg. 1993, I-643, ECLI:EU:C:1992:325, Rn. 21 ff. Parlament/Rat.

148 Valentini (Fn. 39), 7, vgl. 10 m. w. N. 
tiert und unorganisiert erfolgt. ${ }^{149}$ Die Institutionen stimmen sich bei ihrer Öffentlichkeitsarbeit nicht ab, sondern konkurrieren mitunter um Aufmerksamkeit und Positionierung in der Debatte. ${ }^{150}$ Es fragt sich auch, ob der Normappell, der primär verstanden wird als Verpflichtung zu gegenseitiger Rücksichtnabme, ${ }^{151}$ die demokratische Funktion der Öffentlichkeitsarbeit eher schwächt als stärkt. Indem die unterschiedlichen Positionen der EUOrgane untereinander und im Verhältnis zu den Mitgliedstaaten sichtbar werden, wird der Nachrichtenwert des Konflikts bespielt. Solange die einzelnen Akteure den Rahmen ihrer Kompetenzen wahren, sind öffentlich ausgetragene Konflikte, etwa zwischen der Kommission und (Teilen des) Parlaments institutionell verankert und als diskursiver Entscheidungsprozess wünschenswert. Übermäßige Rücksichtnahme oder Kohärenz zu fordern, wäre demgegenüber undemokratisch. Der politische Gestaltungsspielraum wird durch die Loyalitätspflichten deswegen nicht eingeschränkt. ${ }^{152}$ Sie sind im Bereich der Öffentlichkeitsarbeit als bloße Fairness-Regeln zu verstehen, die evidente und anhaltende Störungen sowie die äußersten Grenzen der Kritik untersagen.

\section{Hindernisse und Begünstigungen}

Das Primärrecht wirkt also auf alle drei Qualitätsdimensionen ein. Wenn die beschriebenen Vorgaben beachtet werden, trägt die Öffentlichkeitsarbeit stärker zur demokratischen Legitimation der Europäischen Union bei. Dass sich aus dem Primärrecht Vorgaben an die Qualität ableiten lassen, sagt allerdings noch nichts über deren normative Kraft, ihre tatsächliche Achtung und Befolgung aus. ${ }^{153}$ Das materielle Recht bildet hierbei nur einen Faktor neben organisatorischen, verfahrensrechtlichen und außerrechtlichen, also

149 Brüggemann (Fn. 2), 128, 138; Valentini (Fn. 39), 7, vgl. 10 m. w. N.

150 Valentini (Fn. 39), 6 f. m. w. N.

$151 \mathrm{Zu}$ Art. 13 Abs. 2 S. 2 EUV: Christian Callies, in: Calliess/Ruffert (Fn. 90), Art. 13 EUV Rn. 28; Armin Hatje, in: Jürgen Schwarze, EU-Kommentar, 4. Aufl., 2019, Art. 13 EUV Rn. 34; Carsten Nowak, in: Pechstein/Nowak/Häde (Fn. 99), Art. 13 EUV Rn. 17; zu Art. 4 Abs. 3 EUV; Clandio Franzius, in: Pechstein/Nowak/Häde (Fn. 99), Art. 4 EUV Rn. 169; Stephan Schill/Christoph Krenn, in: Grabitz/Hilf/Nettesheim (Fn. 67), Art. 4 EUV Rn. 126; Christian Callies/ Wolfgang Kabl/Adelheid Puttler, in: Calliess/Ruffert (Fn. 90), Art. 4 EUV Rn. 110.

152 Rudolf. Streinz, in: Streinz (Fn. 96), Art. 13 EUV Rn. 25.

153 Vgl. Konrad Hesse, Die normative Kraft der Verfassung, in: Peter Häberle/Alexander Hollerbach, Konrad Hesse - Ausgewählte Schriften, Heidelberg: C. F. Müller 1984, 12; Konrad Hesse, Grundzüge des Verfassungsrechts der Bundesrepublik Deutschland, 18. Aufl., Heidelberg: C. F. Müller 1991, Rn. 75 f., 42; Otto Depenhener, in: Josef Isensee/Paul Kirchhof (Hrsg.), Handbuch des Staatsrechts, Bd. 12, Heidelberg: C. F. Müller 2014, $\$ 269$ Rn. 1 f. 
sozialen Normen. ${ }^{154}$ Um dysfunktionale Konflikte zu vermeiden, müssen all diese Faktoren ein übergreifendes und in sich stimmiges Konzept ergeben. ${ }^{155}$

Zunächst sollte akzeptiert werden, dass sich das Qualitätsniveau der Öffentlichkeitsarbeit institutionell, kontextuell und situativ stark unterscheidet. Es wäre unangebracht, an die Twitter-Accounts einzelner Parlamentsabgeordneter, die als politische Individuen wahrgenommen werden wollen, und an sich im Rahmen von Interviews frei äußernde Amtsträger dieselben Maßstäbe anzulegen wie an wohlvorbereitete Mitteilungen und Pressekonferenzen des Parlaments, der Kommission oder des Rates. Eine sich zu sehr von den Rahmenbedingungen und Unwägbarkeiten der Massenkommunikation entfernende Idealvorstellung von Öffentlichkeitsarbeit würde sich selbst illegitimieren und ihre handlungsanleitende Kraft einbüßen. ${ }^{156}$ Die nachgelagerten Agenturen sind demgegenüber dem politischen System noch stärker enthoben als die EU-Kollegialorgane und brauchen deswegen noch weniger um individuelle Unterscheidbarkeit bemüht sein. Hier gelten rechtliche Qualitätsvorgaben vergleichsweise rigide.

Der Rechtssicherheit und Außendarstellung wäre es aber zuträglich, wenn Reglements unter der Ebene des Primärrechts aufgestellt würden, welche die Qualitätsvorgaben aktualisieren und konkretisieren. So könnten einzelne EU-Institutionen durch Ausführungsverordnungen (Geschäftsordnungsrecht) die Vorgaben auf ihren jeweiligen Aufgabenbereich, ihre Thematiken und Äußerungsbedingungen - wie schon bzgl. der Transparenzvorgaben (vgl. Art. 15 Abs. 3 UAbs. 3) - näher ausgestalten. Auf diese Weise könnte auch ein für Lernprozesse notwendiges, aber bislang als fehlend gerügtes institutionelles Gedächtnis geschaffen werden. ${ }^{157}$ In diese Richtung zielt der im Sommer 2020 seitens des Parlaments eingerichtete Sonderausschuss zu Einflussnahme aus dem Ausland auf alle demokratischen Prozesse in der Euro-

$154 \mathrm{Vgl}$. Eberhard Schmidt-Aßmann, Das allgemeine Verwaltungsrecht als Ordnungsidee, 2. Aufl., Berlin/Heidelberg, Springer 2004, 245; Christoph Möllers, in: Hans-Heinrich Trute/ Thomas Groß/Hans C. Röhl/Christoph Möllers (Hrsg.), Allgemeines Verwaltungsrecht, 2008, 490; Gunnar F. Schuppert, Grenzen und Alternativen von Steuerung durch Recht, in: Dieter Grimm (Hrsg.), Wachsende Staatsaufgaben - Sinkende Steuerungsfähigkeit des Rechts, BadenBaden: Nomos 1990, 232 ff.; Maximilian Wallerath, Die Änderung der Verwaltungskultur als Reformziel, Die Verwaltung 33 (2000), 351-378 (360).

$155 \mathrm{Vgl}$. Wolfgang Hoffmann-Riem, Regulierung der dualen Rundfunkordnung, BadenBaden: Nomos 2000, 275 f.; Robert Baldwin/Martin Cave/Martin Lodge, Understanding Regulation, 2. Aufl., Oxford: Oxford University Press 2013, $37 \mathrm{f}$.

156 Vgl. Christian Hillgruber, Verfassungsrecht zwischen normativem Anspruch und politischer Wirklichkeit, VVDStRL 67 (2007), 7-56 (10f.); Wolfgang Hoffmann-Riem/Wolfgang Schulz, Politische Kommunikation - Rechtswissenschaftliche Perspektiven, in: Otfried Jarren/ Ulrich Sarcinelli/Ulrich Saxer (Hrsg.), Politische Kommunikation in der demokratischen Gesellschaft, Opladen: Westdeutscher Verlag 1998, 154-172 (161).

157 Vgl. Brüggemann (Fn. 2), 286. 
päischen Union, einschließlich Desinformation, ${ }^{158}$ weil die von diesem entwickelten Qualitätsmaßstäbe letztlich auch Rückschlüsse auf die Güte der eigenen Öffentlichkeitsarbeit zulassen. Relevant sind in diesem Zusammenhang auch eine adäquate personelle und sachliche Ausstattung, die hinreichende Finanzierung der Öffentlichkeitsarbeitsabteilung und die zeitgemäße Aus- und Fortbildung der dort beschäftigten Personen. ${ }^{159}$

Doch unabhängig davon, auf welcher Ebene des Unionsrechts Vorgaben getroffen werden, sind diese gerichtlich nicht optimal durchsetzbar. Die rechtswirksamkeitssteigernden Funktionen der Judikative kommen für Öffentlichkeitsarbeit nicht vollends zur Geltung. Generell ist der gerichtliche Rechtsschutz auf Unionsebene mit den Verfahrensarten vor dem EuGH und dem EuG nicht auf rein informelles beziehungsweise informationelles Handeln ausgelegt. ${ }^{160}$ Für Öffentlichkeitsarbeit kommt hinzu, dass diese oftmals keine subjektiven Rechte ${ }^{161}$ Dritter berührt, sondern lediglich auf gesellschaftlich-demokratischer Ebene wirkt. Damit treten anderweitige Kontrollmechanismen auf den Plan. Vorstellbar wäre etwa ein jährlicher Bericht über die unionsweite Qualität der Öffentlichkeitsarbeit, ähnlich des Berichts der Kommission über die Lage der Rechtsstaatlichkeit in der Europäischen Union. ${ }^{162}$ Es könnte aber etwa auch der Europäische Bürgerbeauftragte zu verstärkten Regulierungs- und Evaluationsbemühungen angehalten werden. Er verfügt über Expertise, hat er doch den Kodex für gute Verwaltungspraxis und die Grundsätze des öffentlichen Dienstes für EU-Beamte ausgearbeitet. ${ }^{163}$ Mit der Beschwerde zum Europäischen Bürgerbeauftragten gem. Art. 228 AEUV besteht bereits ein formloser Rechtsbehelf, mit dem die Öffentlichkeitsarbeit gerügt werden kann, ohne dass man durch diese unmittelbar betroffen sein muss. ${ }^{164}$ Eine herausgehobene Kontrollfunktion kommt indes den Rezipienten als kritischer Öffentlichkeit zu. Als originäre Nutznießer qualitativ hochwertiger Öffentlichkeitsarbeit obliegt es ihnen, eine solche auch einzufordern. Zwar wird sich zivilgesellschaftliche

158 2020/2683(RSO).

159 Meyer (Fn. 2), 623, $628 \mathrm{f}$.

160 Siehe Timo Rademacher, Realakte im Rechtsschutzsystem der Europäischen Union, Tübingen: Mohr Siebeck 2014, passim.

161 Vgl. Art. 47 Abs. 1 GrC: „Jede Person, deren durch das Recht der Union garantierte Rechte oder Freiheiten verletzt worden sind [...]“.

$162 \mathrm{Im}$ erstmals erschienen Jahresbericht der Kommission (COM(2020) 580 final) wird unter Nr. 2.3 die Bedeutung der Medienfreiheit und des Medienpluralismus hervorgehoben. Siehe auch die auf Grundlage des Art. 295 S. 2 AEUV erfolgte Entschließung des Europäischen Parlaments vom 25. Oktober 2016 (2015/2254(INL)); hierzu Möllers/Schneider (Fn. 23), $109 \mathrm{ff}$.

163 Vgl. Siegfried Magiera, in: Meyer/Hölscheidt (Fn. 80), Art. 41 Rn. 16.

164 Vgl. Winfried Kluth, in: Calliess/Ruffert (Fn. 90), Art. 228 AEUV Rn. 9; ausführlich zum Institut Oliver Mader, Bürgerinitiative, Petitionsrecht, Beschwerde zum Bürgerbeauftragten, EuR 48 (2013), 348-371 (364 ff.). 
Kritik nicht an den Vorgaben des Primärrechts orientieren, sondern intuitivere Vorstellungen guter Öffentlichkeitsarbeit zum Maßstab wählen. Dennoch kann der Grad der Akzeptanz der Europäische Union den Organen dazu dienen, sich ihrer Rolle zu vergewissern und Anpassungsprozesse anzustoßen. ${ }^{165}$

\section{Puzzlestück statt Allheilmittel}

Man sollte von demokratisch und rechtsstaatlich ausgerichteter Öffentlichkeitsarbeit, so gut sie auch gestaltet sein mag, keine Wunder erwarten. Sie wird aus eigener Kraft keine einheitliche europäische Öffentlichkeit schaffen und die Zugehörigkeit und Identifikation der Unionsbürger mit der Europäischen Union nicht auf Anhieb spürbar verbessern können. Auch Nationalstaaten stehen insoweit trotz leichterer Rahmenbedingungen vor teils gravierenden Problemen. ${ }^{166}$ Das supranationale Institutionengeflecht der Europäischen Union erschwert die Kommunikation demgegenüber. Dass die Auflagen der europäischen Broschüren, die Follower- und Like-Zahlen der EUOrgane verglichen mit der knappen halben Milliarde an Unionsbürgern meist marginal ausfallen, hängt deswegen sicherlich nicht nur damit zusammen, dass die Europäische Union bislang noch nicht die richtige Kommunikationsstrategie gefunden hätte. Und doch kann sie sich diesen widrigen Umständen nicht einfach geschlagen geben. Sie hat in der guten Öffentlichkeitsarbeit ein unabdingbares Puzzlestück, und damit eine weitere Herausforderung auf ihrem Weg der Verwirklichung einer immer engeren Union gefunden. Gute Öffentlichkeitsarbeit stellt keine hinreichende, aber eine notwendige Bedingung lebendiger Öffentlichkeit dar und stärkt die demokratische Legitimation der Europäischen Union. Die Regelungen der EUV, AEUV und GrC bilden Fundament und Grenze für diese. Sie untersagen allzu vereinfachende und auf Marketing bedachte Strategien, die vielleicht kurzfristig Erfolg versprechen, aber langfristig die Vertrauenswürdigkeit europäischer Öffentlichkeitsarbeit untergraben würden. Wie stark ihre handlungsanleitende Kraft tatsächlich ausfällt, hängt davon ab, ob aus ihnen spezifische Informationspolitiken konkretisiert, ob sie in eine adäquate institutionelle und prozedurale Struktur gebettet und von entsprechenden sozialen Normen unterstützt werden.

165 Vgl. Mast (Fn. 71), $401 \mathrm{f}$.

166 von Danwitz (Fn. 113), 44. 


\section{Summary: Good Public Relations and the European Union}

The European Union is not a state, yet it is founded on the values of democracy and the rule of law. It is therefore astonishing that scholars of European law have so far hardly dealt with the public relations of the European Union, although they question its democratic legitimacy. In terms of its institutional and legal framework, the European Union's public relations differs substantially from that of its member states. This article presents findings from communication and political science on the deficits of the current public relations work of the European Union. Based on these findings it works out to what extent Primary Law can counteract these deficits.

\section{Keywords}

European Union - public relations - democracy principle 